Stavros A. Savidis, Daniel Aubram, Frank Rackwitz

\title{
Vibro-Injection Pile Installation in Sand: Part II-Numerical and Experimental Investigation
}

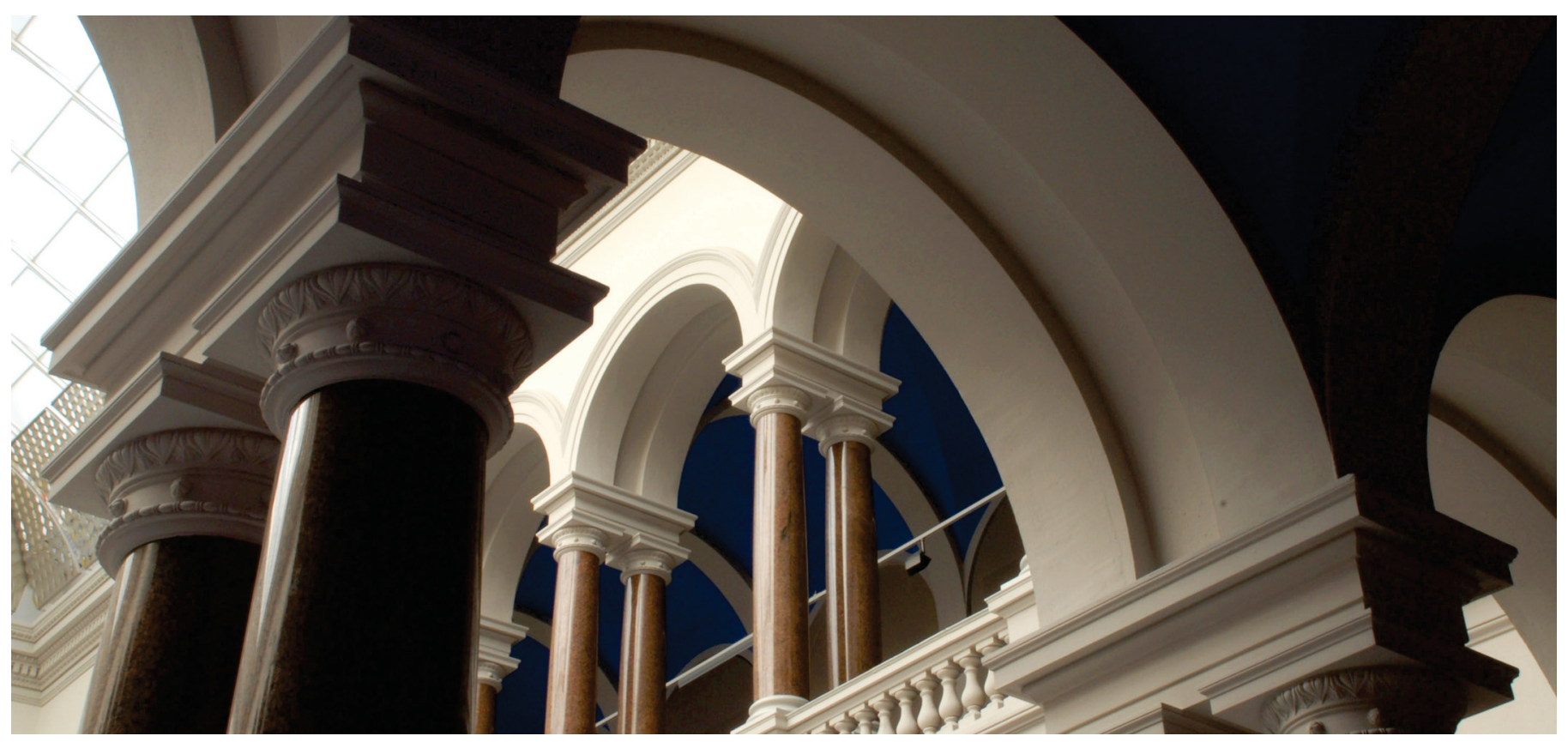

Savidis S.A., Aubram D., Rackwitz F. (2015) Vibro-Injection Pile Installation in Sand: Part II-Numerical and Experimental Investigation. In: Triantafyllidis T. (eds) Holistic Simulation of Geotechnical Installation Processes. Lecture Notes in Applied and Computational Mechanics, vol 77. Springer, Cham. pp. 103-131. https://doi.org/10.1007/978-3-319-18170-7_6 


\title{
Vibro-Injection Pile Installation in Sand: 2. Numerical and Experimental Investigation
}

\author{
Stavros A. Savidis ${ }^{1}$, Daniel Aubram ${ }^{1}$, and Frank Rackwitz ${ }^{2}$ \\ 1 Chair of Soil Mechanics and Geotechnical Engineering, Technische Universität \\ Berlin, Secr. TIB1-B7, Gustav-Meyer-Allee 25, D-13355 Berlin, Germany \\ 2 Department of Geotechnical Engineering, Ostbayerische Technische Hochschule \\ Regensburg, Prüfeninger Straße 58, D-93049 Regensburg, Germany
}

\begin{abstract}
In Part 1 of this series of papers a macroscopic two-equation (two-field) reduced model for the mechanics of the multi-material flow associated with vibro-injection pile installation in saturated sand was derived. Here we employ this model to develop a so-called multi-material arbitrary Lagrangian-Eulerian (MMALE) method. MMALE avoids the disadvantages of the classical approaches in computational continuum mechanics concerning large deformations and evolving material interfaces. The numerical implementation of this method will be outlined, and then the experimental investigations will be presented that have been carried out in order to validate the computational model. Among these investigations, small-scale model tests in chambers with observing window have been designed step-by-step to reveal penetration and vibro-injection pile installation phenomena.
\end{abstract}

Keywords: arbitrary Lagrangian-Eulerian, multi-material, large deformations, finite element method, operator-split, soil mechanics, sand

\section{Introduction}

Subproject 5 as part of the DFG Research Unit FOR 1136 is concerned with the numerical modeling of vibro-injection pile installation into water-saturated sand. The motivation arises from the fact that there are currently no numerical models to accurately predict the effects of this process on the stress and density states within the soil or on close-by structures. However, such a prediction would be of high practical relevance. In Part 1 of our contribution we interpreted the process of vibro-injection pile installation as the flow of an immiscible mixture with interfaces (multi-material flow) consisting of a bulk solid phase (steel pile), a bulk fluid phase (injection grout), and a compound phase consisting of a solid species and a fluid species which represents the fluid-saturated porous material (sand). Application of the technique of spatial averaging to the governing equations led to a macroscopic two-equation (two-field) model. In Part 2 we continue our investigation and employ this continuum mechanical model to develop a multimaterial arbitrary Lagrangian-Eulerian (MMALE) method for the numerical simulation of vibro-injection pile installation.

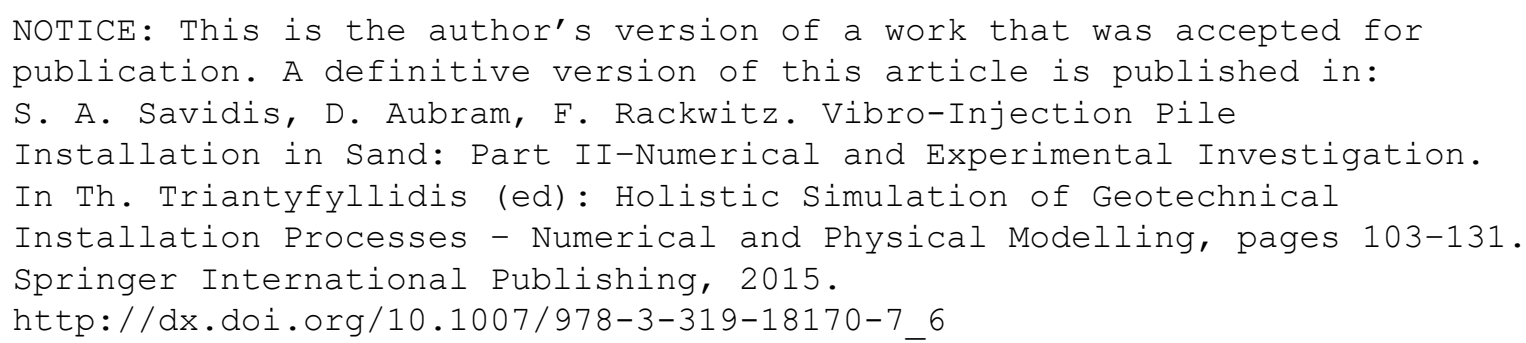


MMALE falls into the category of arbitrary Lagrangian-Eulerian (ALE) numerical methods in which the mesh is not fixed as in the Eulerian methods but can move independently of the material [30, 49, 8, 6]. MMALE methods, including the subset of multi-material Eulerian methods, generalize the classical approaches according to Lagrange and Euler in the context of finite element or finite difference methods and are able to address the problems mentioned $[20,61,37,38,11,12,13,14,19,39,44,56,24]$. No limitations concerning material deformations do exist because in these methods material boundaries may flow through the mesh. Therefore, elements may arise which contain two or more materials separated by interfaces.

Both MMALE and the subset of multi-material Eulerian methods were originally developed in the defence sector for the numerical simulation of highly dynamical physical problems in which high strain rates appear and new interfaces are generated (hypervelocity multi-material flow). Information is often hard to access because of the classification restriction imposed on many program codes and the related developments. Typical application areas are underwater explosions with fluid-structure-interaction and impact problems. However, with the application of MMALE methods to water-saturated sand at relatively low velocities this subproject has entered new territory on both national and international level.

The change from the Lagrangian to a non-Lagrangian viewpoint inherent to MMALE methods introduces three problems: (i) tracking material interfaces, (ii) treatment of multi-material elements intersected by interfaces, and (iii) advection of the solution variables across element boundaries. Due to the potential presence of multiple materials in a single element, MMALE requires the solution of a multi-material flow problem in consideration of solid mechanical, fluid dynamical, and interaction phenomena. Consequently, the theoretical basis includes aspects of both continuum mechanics and multiphase flow theory. The numerical treatment, on the other hand, requires FEM technology as well as methods from the field of computational fluid dynamics (CFD). This renders MMALE highly interdisciplinary and complex, as visualized in Fig. 1.

The mixture of multiple materials in multi-material elements is treated as an effective single-phase material or homogenized mixture on the element level; empty space (void) is considered as a type of material having vanishing stiffness and mass density. The degrees of freedom of the homogenized mixture solved for at the element nodes are the same for all individual phases of the mixture. In the present model all phases share a common velocity and pressure. Such an approach calls for appropriate mixing rules which relate quantities associated with each material to the corresponding "mixed" (homogenized) variables, and vice versa. The mixing rules play a crucial role and should be governed by the physics of the problem. In particular, the saturated sand in vibro-injection pile installation must be modeled as a two-phase material in order to account for consolidation and liquefaction phenomena. During the remap step of the method the partial material volumes in multi-material elements must be transported through the mesh. In order to achieve a reasonable accuracy of the procedure, the 


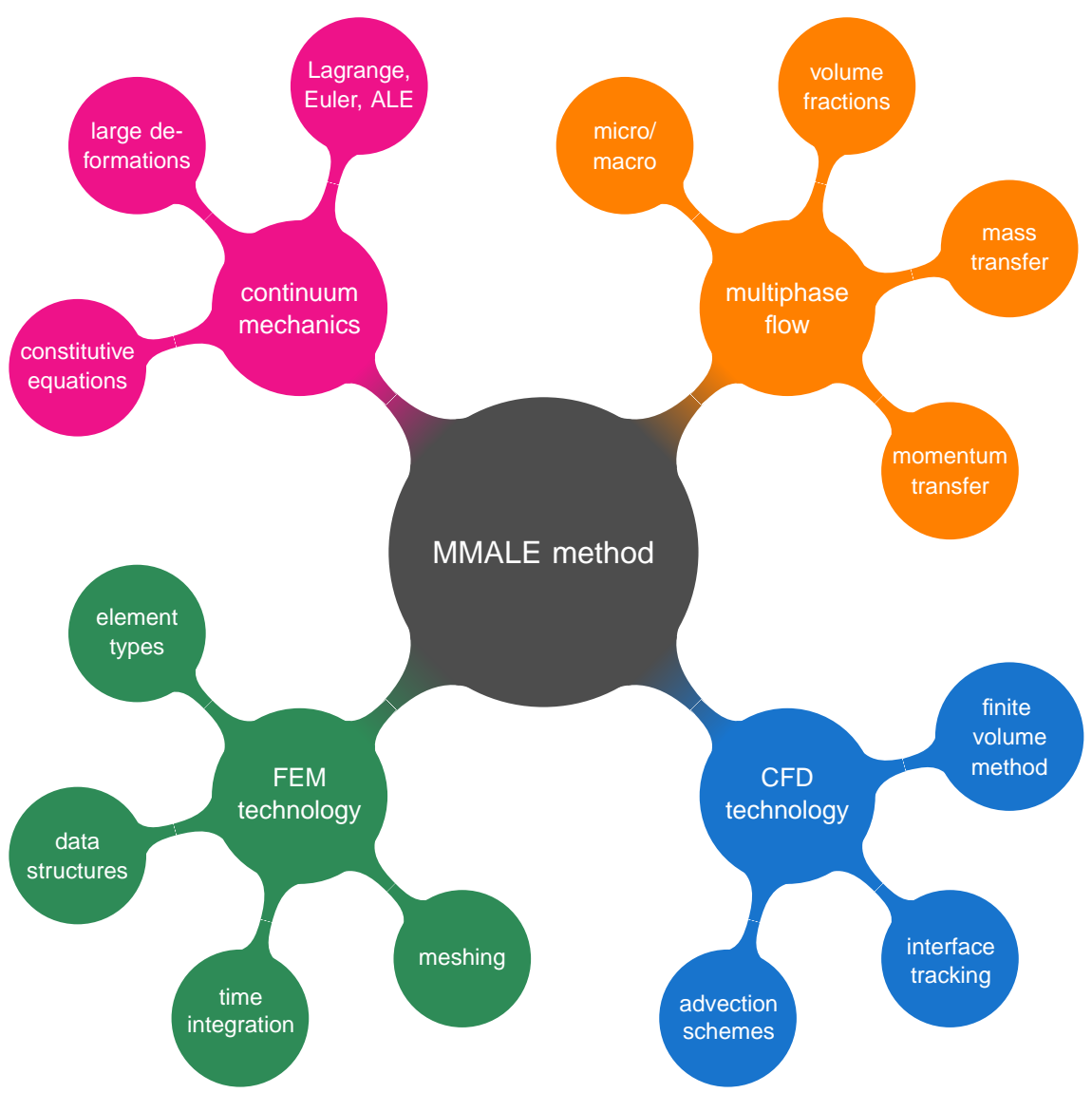

Fig. 1. Mind map of the developed MMALE method to visualize its interdisciplinarity and complexity. 
material volume fluxes across the element boundaries are computed as truncation volumes which requires material interfaces to be tracked along with the flow field.

Part 2 in this series of papers is structured as follows. The development and implementation of the MMALE computational method will be outlined in Section 2. It consists of several algorithms specific to multi-material methods which will be briefly addressed. The conception and realization of a model test chamber with observing window and the conducted experimental small-scale tests will be described in Section 3. These experimental investigations are undertaken supplementary to the central project of this research unit in order to validate the MMALE method. Section 4 presents example applications of the numerical algorithms as well as preliminary results of back-calculations. The paper closes with concluding remarks and outlook in Section 5 .

\section{Numerical Method and Implementation}

\subsection{ALE Formulation and Solution Strategy}

The governing equations of the two-equation (two-field) reduced model derived in Part 1,

$$
\begin{aligned}
\operatorname{div}(\boldsymbol{s}-p \boldsymbol{I})+\rho \boldsymbol{b}-\rho \dot{\boldsymbol{v}} & =\mathbf{0} \\
\dot{p} / K+\operatorname{div} \boldsymbol{v} & =0,
\end{aligned}
$$

are in the so-called updated Lagrangian form, meaning that the current configuration of the material in space is taken as the reference domain deforming with the material as time elapses. The entire model has been formulated by Eqs. (63)-(68) in that paper. Herein we consider only averaged quantities, so that the angle brackets $\langle\cdot\rangle$ can be dropped for reasons of notational brevity. The basic notation is found in Part 1.

Since the reference domain is represented by the finite element mesh in numerical implementations, large material deformations may cause severe distortion of Lagrangian elements which slows down or even terminates the calculation. The ALE formulation has been developed in order to circumvent these problems by introducing an independently moving reference domain [5, 6]. Accordingly, the spatial description of any scalar-, vector- or tensor-valued physical field $q$ is related to its referential or ALE description $\hat{q}$ by the composition $\hat{q}=q \circ \Phi$, where $\Phi$, called the relative motion, is an embedding that maps the reference points onto the spatial points currently occupied by the material.

The material time derivative of $q=\hat{q} \circ \Phi^{-1}$ leads to the fundamental ALE operator

$$
\dot{q}=\frac{\partial \hat{q}}{\partial t} \circ \Phi^{-1}+c \cdot \nabla q, \quad \text { with } \quad \dot{q} \stackrel{\text { def }}{=} h(\ldots),
$$

and $h(\ldots)$ representing an evolution equation for the field $q$ under consideration. The first term on the right side represents the time derivative of $q$ with respect to fixed reference points. The second term, called the convective term, stems from the relative motion between the material and the reference domain defining the so-called convective velocity $\boldsymbol{c}$ for each material; as we assumed homogeneous 
distribution of velocity in the mixture, $\boldsymbol{c}$ is the same for all constituents. Note that (2) generalizes the classical Lagrangian $(\boldsymbol{c}=\mathbf{0} ; \Phi=$ motion of the body) and Eulerian $(\boldsymbol{c}=\boldsymbol{v} ; \Phi=$ id) formulations of continuum mechanics. On the other hand, replacing with the ALE operator (2) every material time derivative in the derived model for multi-material flow results in an ALE formulation of that model which can be treated by an MMALE method. The resulting system of equations can be brought into a convenient conservation form [6], but this is not shown here.

The MMALE method developed in this research work is an extension of the simplified or single-material ALE method $[49,8,6]$ in which material interfaces are explicitly resolved by element edges. An operator-split or Lagrange-remap strategy $[11,6]$ is applied to enable the use of simpler and more robust algorithms compared to a monolithic solution approach. It divides the incremental solution of the highly nonlinear problem into a Lagrangian step and remap step, as schematically shown in Fig. 2. Concerning the fundamental ALE operator (2), the Lagrange-remap strategy can be written conceptually as

$$
\begin{aligned}
\text { Lagrangian step: } & \dot{q}=h(\ldots), \\
\text { remap step: } & \frac{\partial \hat{q}}{\partial t} \circ \Phi^{-1}+\boldsymbol{c} \cdot \nabla q=0 .
\end{aligned}
$$

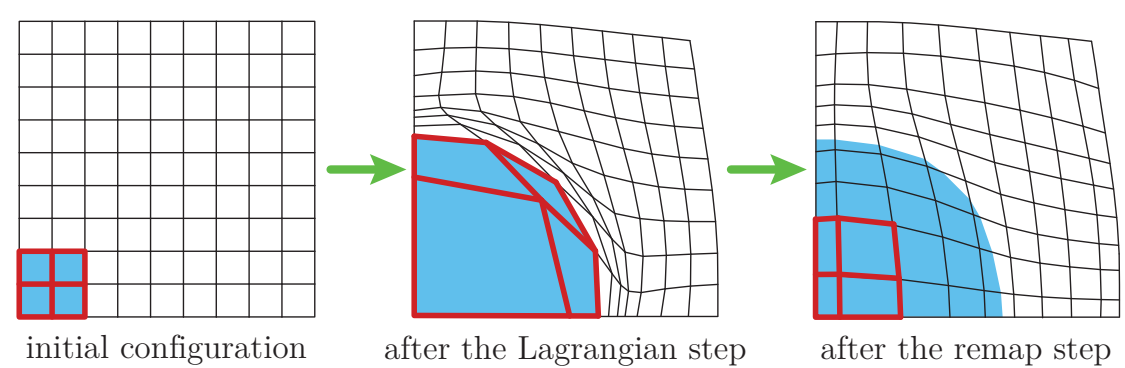

Fig. 2. Schematic diagram of the Lagrange-remap MMALE method. The blue area indicates a material zone whose initial configuration is assigned to an element patch highlighted in red.

Equation (3) represents the updated Lagrangian form of the equations (63)(68) in Part 1 governing the considered multi-material flow of vibro-injection pile installation into water-saturated sand. During the Lagrangian step the set of equations is solved with common finite element methods for the two-field mixed element formulation by accounting for large deformations $[36,62,63,10,60]$. For this purpose the system of equations (1) is brought into a weak form, and then this weak form is approximated by discretizing the computational domain. The so-called MINI or $\mathrm{P} 1^{+} / \mathrm{P} 1$ element $[3,18]$ serves as a basis for the developments. It is a mixed triangle element using linear approximations for the spatial pressure 
field $p$ and spatial displacement field $\boldsymbol{u}$, with $\dot{\boldsymbol{u}}=\boldsymbol{v}$. An additional bubble function for the displacement field stabilizes the element. The MINI Element is equipped with a multi-material option during the research project for the purposes of MMALE implementation (Fig. 3).

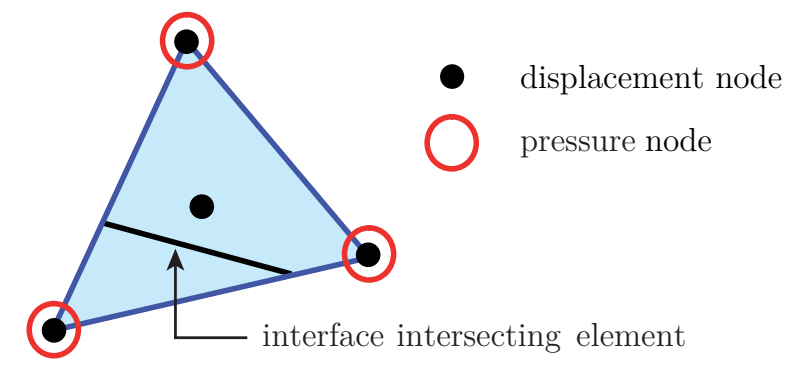

Fig. 3. An essential part of the MMALE method is the stable MINI element [3] which is enriched during this research by a multi-material option in order to account for interfaces intersecting the element.

In the Lagrangian step the parametrization of the variables of the problem is chosen such that the element mesh follows the deformation of the mixture and the convective terms disappear. The evolution equations of the variables take the form of (3). That is to say, the Lagrangian step considers the sources, constitutive equations of each material, and topological closure laws for each phase as in standard nonlinear finite element analysis. The remap step accounts for the convective terms by solving (4), which represents the linear advection equation without a source. For this purpose, the nodes are relocated in such a way that mesh distortion is reduced and then the solution variables obtained after the Lagrangian step are transported through the mesh using conservative CFD advection algorithms. If the nodes are simply relocated to their original positions a multi-material Eulerian formulation is obtained. In this case, (4) reduces to the well-known linear advection equation without a source:

$$
\frac{\partial q}{\partial t}+\boldsymbol{v} \cdot \nabla q=0
$$

A flow chart of the developed MMALE method is shown in Fig. 4. The program steps will be explained in the following.

\subsection{Initialization Phase}

Before the actual calculation starts the computational domain of the considered initial boundary value problem is discretized with finite elements. Depending on the problem definition, the computational domain has to cover empty space which might be occupied by material during the course of the calculation. Empty space (void) is considered as a type of material within the model; see constitutive 


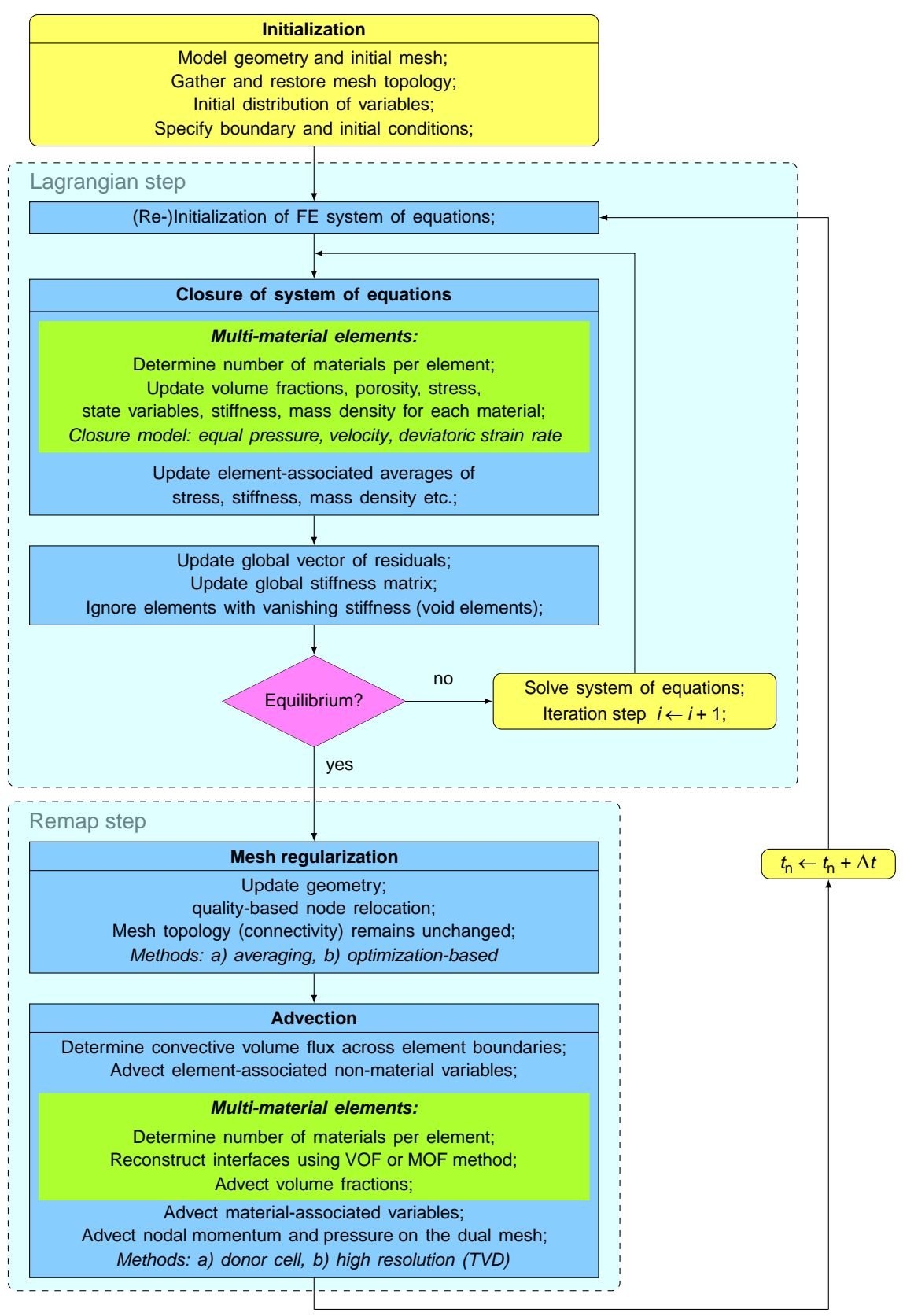

Fig. 4. Flow chart of the developed MMALE method for finite element programs. 
equations summarized as equation (65) in Part 1. The remap step requires additional information about the mesh connectivity, such as the elements sharing a common node or the adjacent elements of an element. However, this information has to be gathered and stored only once during the initialization phase because the mesh connectivity, by definition, does not change in ALE methods. Furthermore, the material properties must be assigned to the elements. The initialization phase is completed with the specification of the initial conditions and boundary conditions.

\subsection{Lagrangian Step}

A calculational cycle or time increment begins in the Lagrangian step with the re-initialization of the FE system of equations. Solution is advanced implicitly in time by using the Newmark- $\beta$ method in conjunction with Newton's method. This time integrator is unconditionally stable, hence imposes no restriction on the size of the time step in contrast to the explicit procedures applied in almost all other multi-material Eulerian and MMALE methods. However, the system of equations must be delivered in linearized form which generally requires the determination of the geometric stiffness matrix of each element and of the material tangent being consistent with the stress integration procedure, and not simply the continuum tangent $[10,60]$. However, these are not essential for problem solution but at most influence the rate of solution convergence. Therefore, in order to simplify numerical implementation the element stiffness matrix is derived from the continuum material tangent alone by neglecting the geometric stiffness matrix, as this has been done in [14].

The treatment of multi-material elements in the Lagrangian step makes use of the subcell model derived in Part 1 and starts with the determination of the number of materials in each element. Afterwards the state of each material phase is updated. In particular, the volume fraction, porosity, stress, state variables, and material tangent of each material are updated by integrating the related evolution equations in time. The rotational terms of the stress rate at finite deformation render the integration of rate constitutive equations expensive compared to the infinitesimal case. In this context, the restrictions related to the axiom of material frame indifference [54] have led to the notion of incremental objectivity of the integration method over finite time steps [31]. Incremental objectivity requires that if the motion of the material body over a time increment $\Delta t=t_{n+1}-t_{n}$ is rigid, then the stress is exactly updated without generation of spurious stresses. The same is required for tensor-valued material state variables.

The stress integration in the Lagrangian step of the MMALE method employs the incrementally objective algorithm of [32]. Accordingly, the stress integration is carried out with respect to material points in the corotated (unrotated) configuration of the material body. A time-centered approximation over the incremental time interval $\left[t_{n}, t_{n+1}\right]$ is used in accordance with [31], through which 
algorithmic finite strain and rotation increments are computed as

$$
\begin{aligned}
& \Delta \boldsymbol{\varepsilon}_{n+1 / 2} \stackrel{\text { def }}{=} \boldsymbol{d}_{n+1 / 2} \Delta t=\frac{1}{2}\left(\boldsymbol{\nabla}_{n+1 / 2} \boldsymbol{u}+\left(\boldsymbol{\nabla}_{n+1 / 2} \boldsymbol{u}\right)^{\mathrm{T}}\right) \quad \text { and } \\
& \Delta \boldsymbol{r}_{n+1 / 2} \stackrel{\text { def }}{=} \boldsymbol{\omega}_{n+1 / 2} \Delta t=\frac{1}{2}\left(\nabla_{n+1 / 2} \boldsymbol{u}-\left(\boldsymbol{\nabla}_{n+1 / 2} \boldsymbol{u}\right)^{\mathrm{T}}\right),
\end{aligned}
$$

respectively, and

$$
\boldsymbol{\nabla}_{n+1 / 2} \boldsymbol{u}=2\left(\boldsymbol{f}_{n+1}-\boldsymbol{I}\right)\left(\boldsymbol{f}_{n+1}+\boldsymbol{I}\right)^{-1} .
$$

$\boldsymbol{f}_{n+1}$ is the relative incremental deformation gradient of the configuration at time $t=t_{n+1}$ with respect to the configuration at time $t=t_{n}$.

Now, consider the general rate constitutive equation

$$
\stackrel{\nabla}{\sigma}=\boldsymbol{c}(\boldsymbol{\sigma}, \boldsymbol{\alpha}): \boldsymbol{d}
$$

where $\stackrel{\nabla}{\boldsymbol{\sigma}}=\dot{\boldsymbol{\sigma}}+\boldsymbol{\sigma} \cdot \boldsymbol{\omega}-\boldsymbol{\omega} \cdot \boldsymbol{\sigma}$ denotes the corotational Zaremba-Jaumann rate of $\boldsymbol{\sigma}$ defined by the spin $\boldsymbol{\omega}$ representing the vorticity tensor. Since the spin generates a one-parameter group of rotations through $\dot{\mathfrak{R}}=\boldsymbol{\omega} \cdot \mathfrak{R}$, with $\left.\mathfrak{R}\right|_{t=0}=\boldsymbol{I}$, the Cauchy stress is objectively updated by the general integration algorithm

$$
\boldsymbol{\sigma}_{n+1}=\mathfrak{R}_{n+1} \cdot\left(\mathfrak{S}_{n}+\Delta \mathfrak{S}_{n+\theta}\right) \cdot \mathfrak{R}_{n+1}^{\mathrm{T}}, \quad \text { with } \quad \theta \in[0,1],
$$

and

$$
\begin{array}{r}
\mathfrak{S}_{n} \stackrel{\text { def }}{=} \boldsymbol{\Re}_{n}^{\mathrm{T}} \cdot \boldsymbol{\sigma}_{n} \cdot \boldsymbol{\Re}_{n}, \quad \Delta \mathfrak{S}_{n+\theta} \stackrel{\text { def }}{=} \mathfrak{f}_{n+\theta}\left(\mathfrak{S}_{n+\theta}, \mathfrak{A}_{n+\theta}, \Delta \mathfrak{E}_{n+1 / 2}\right), \\
\Delta \mathfrak{E}_{n+1 / 2} \stackrel{\text { def }}{=} \mathfrak{R}_{n+1 / 2}^{\mathrm{T}} \cdot \Delta \boldsymbol{\varepsilon}_{n+1 / 2} \cdot \mathfrak{R}_{n+1 / 2}, \quad \boldsymbol{S}_{n+\theta} \stackrel{\text { def }}{=} \boldsymbol{\Re}_{n+\theta}^{\mathrm{T}} \cdot \boldsymbol{\sigma}_{n+\theta} \cdot \mathfrak{R}_{n+\theta},
\end{array}
$$

and $\boldsymbol{\mathfrak { A }}_{n+\theta}$ representing the set $\boldsymbol{\alpha}$ of material state variables under the transformation $\mathfrak{R}_{n+\theta}$. The stress increment $\Delta \boldsymbol{S}_{n+\theta}$ is calculated using the response function $\mathfrak{f}_{n+\theta}$. The response function depends on the choice of $\theta \in[0,1]$ and basically represents an explicit $(\theta=0)$ or implicit $(\theta=1)$ stress-point algorithm for the case of infinitesimal deformations. The rotation and half-step rotation are defined through

$$
\mathfrak{R}_{n+1}=\Delta \mathfrak{R} \cdot \mathfrak{R}_{n} \quad \text { and } \quad \boldsymbol{R}_{n+1 / 2}=\Delta \mathfrak{R}^{1 / 2} \cdot \mathfrak{R}_{n}
$$

where

$$
\Delta \mathfrak{R}=\left(\boldsymbol{I}-\frac{1}{2} \Delta \boldsymbol{r}_{n+1 / 2}\right)^{-1}\left(\boldsymbol{I}+\frac{1}{2} \Delta \boldsymbol{r}_{n+1 / 2}\right)
$$

is an approximation to the incremental rotation over the time increment $\left[t_{n}, t_{n+1}\right]$ according to [31]. If the configuration at time $t=t_{n}$ is taken as the reference configuration, as in an updated Lagrangian description of motion [10, 6], then $\boldsymbol{R}_{n}=\boldsymbol{I}$, otherwise $\boldsymbol{R}_{t=0}=\boldsymbol{I}$ is set.

Algorithm (9) is applied to each material phase whose mechanical behavior is characterized by a rate constitutive equation of the form (8). According to (52) in Part 1, this would be the case for bulk solid and porous media. In particular, the behavior of sand is described by an advanced hypoplastic rate constitutive equation $[57,40]$. This constitutive equation is advanced in Subproject 1 
of the Research Unit FOR 1136. For a reliable numerical simulation of vibroinjection pile installation it is indispensable to carry over new developments to the MMALE method.

After the material-associated variables were updated, their element-associated averaged values can be determined in accordance with (64) in Part 1 . The balance between the internal forces and the external loads at the end of the time step, which expresses the identity of the vector of residuals with the null vector, is iterated by means of Newton's method. Elements that partially or completely cover empty space require special treatment in implicit multi-material Eulerian and MMALE calculations [14]. Void elements practically do not have any stiffness or mass density. Therefore, their nodes remain unconsidered when setting up the finite element system of equations. Elements located at material boundaries are partially filled with void. The stiffness of the mixture inside these elements might be low, causing large displacement increments during the equilibrium iterations. Therefore, in order to avoid inverted elements, the incremental nodal displacements of partially filled elements are uniformly scaled.

\subsection{Remap Step}

When the equilibrium iteration converges, the quality of all elements in the mesh is evaluated. The quality measure employs the radius ratio of the element's incircle and circumcircle. The remap step is initialized if at least one element fails the quality check, and then the nodes of those elements are flagged. Only the flagged nodes and the elements that share these nodes are processed during the remap step for reasons of computational efficiency. Therefore, all algorithms of the remap step are required to work on a local level.

After the initialization of the remap step the geometry of the FE model is updated, so that the totality of nodes in their current position defines the reference domain. In order to increase the quality of the mesh deformed during the Lagrangian step, the flagged nodes are relocated by employing a suitable local mesh smoothing algorithm. Users can choose between different heuristic procedures and an extremely robust optimization-based smoothing algorithm which works on arbitrarily shaped domains [6, 7]. A multi-material Eulerian formulation ( $\boldsymbol{c}=\boldsymbol{v} ; \Phi=\mathrm{id})$ is obtained if the nodes are simply relocated to their original positions.

One of the crucial and at the same time most extensive steps of the MMALE method is the transport (advection) of the variables through the mesh. Technically speaking this means a remap of the solution variables obtained after the Lagrangian step onto the smoothed mesh. Because the mesh topology does not change during the smoothing step, elements have the same neighbors throughout the calculation, so that conservative CFD algorithms can be applied. The remap must be carried out for element-associated variables (e.g. deformation gradient), material-associated variables (e.g. stress and state variables), and nodal variables. The latter are dictated by the primary unknowns of the problem and the underlying balance equations. Concerning the two-equation model considered 
here (see Part 1), the nodal variables are represented by the total momentum and total volume change of the mixture.

For unstructured meshes, transport algorithms which are based on the finite volume method [35, 9] are best suited. Finite volume (FV) methods solve the integral form of the advection equation (4) as an integral conservation law. Hence, they are conservative by definition, leading to mechanically consistent results. In order to solve the integral conservation law for the generic variable $q$ under consideration, a control volume tessellation has to be constructed based on the finite element mesh that is used in the overall MMALE solution procedure. Moreover, a pseudo-time interval $\left[t^{-}, t^{+}\right] \subset \mathbb{R}$ is introduced because physical time elapses during the Lagrangian step but remains fixed during the remap step. The distribution of the variables at the end of the Lagrangian step $\left(t=t^{-}\right)$is assumed to be given, that is, $\left.q\right|_{t=t^{-}}=q^{-}$. Then, if a first-order accurate integration in time is applied, the variable in the $j$-th control volume after the remap step at $t=t^{+}$is obtained by the general formula

$$
q_{j}^{+}=\frac{q_{j}^{-} V_{j}^{-}-\sum_{\mathrm{facets}} \operatorname{Fl}(q) \Delta t}{V_{j}^{+}} .
$$

Here $V_{j}$ and $q_{j}$ denote the volume measure and the average of $q$ associated with the $j$-th control volume, respectively, $\operatorname{Fl}(q)$ is the averaged convective volume flux of $q$ across a facet of the control volume boundary, and $\Delta t \stackrel{\text { def }}{=} t^{+}-t^{-}$. The control volume geometries to compute $V_{j}^{-}$and $V_{j}^{+}$can be determined from the mesh geometries known at $t=t^{-}$and $t=t^{+}$, respectively.

Inevitably connected with FV methods is the computation of the flux of a solution variable across the boundary of the control volumes; cf. (13). Depending on the accuracy in space of the scheme used for the flux calculation, advection algorithms are divided into first-order, second-order, and higher-order accurate methods. Moreover, the flux of a solution variable can be a linear or nonlinear function of the transported volume, leading to a linear or nonlinear procedure. Despite this, most finite volume advection schemes in ALE methods apply explicit first-order accurate methods to advance solution in time.

A weighted donor-cell linear advection scheme [30, 4, 47, 11, 44, 6] of the Godunov-type is currently implemented. It possesses an accuracy of first order and is stable, conservative, and monotonicity-preserving. The linear procedures of first-order are in fact the most robust and the easiest to implement, but they tend to excessive numerical diffusion through which solution details are smeared. Though with linear schemes of higher order this tendency is less pronounced, the solution can oscillate. Modern nonlinear algorithms can avoid spurious oscillation and at the same time achieve a maximum accuracy of second or higher order in space. The state of the art in the field of MMALE are high-resolution nonlinear schemes, for example total variation diminishing (TVD) algorithms [27, 11, 35, 24]. Such an algorithm will be also implemented into the developed MMALE method.

Concerning multi-material elements cut by one or more non-intersecting interfaces, material-associated variables must be treated separately for each in- 
dividual material. Accordingly, the field to be used in (2)-(4) is the materialassociated variable weighted by the volume fraction, i.e. $q \stackrel{\text { def }}{=} f^{k} q^{k}$. If one would simply use this definition in the general finite volume-based transport algorithm (13), however, the initially coherent material phases would disperse after a few advection steps because the interfacial structure is not accounted for [24].

In order to achieve a reasonable accuracy of the remap procedure and to conserve the volume of the individual phases, the volume fractions and the materialassociated fields have to be transported by considering the spatial distribution of the material phases and the locations of the interfaces in every multi-material element. This requires specialized methods to resolve material interfaces during an MMALE or multi-material Eulerian calculation. Such methods, which can be divided into interface tracking and interface reconstruction methods, have been reviewed in $[33,48,46,50,15]$.

Interface tracking methods update the locations of the material interfaces at each time step. Basic approaches use Lagrangian marker particles [25, 26, $55]$ or level set functions $[42,52,43]$. Both approaches are extensively used in CFD and computer graphics but they are prone to numerical difficulties when the interfaces experience severe stretching or tearing. In particular, level set methods do not locally conserve volume. Interface reconstruction methods like the volume of fluid (VOF) [29, 61, 45] and moment of fluid (MOF) methods [22, $23]$, on the other hand, are generally conservative because they track the volume resp. moment (i.e. volume and centroid) of a partial material zone (subcell) in multi-material elements.

Once the interface locations in each multi-material element have been determined by any of the methods mentioned, the material transport volumes across the element facets can be computed as truncation volumes, and then the partial material volumes are integrated to a new time level. The actual calculation is largely geometrical in nature and includes basic algorithms like the point-inpolygon test, intersection tests, area computation, and clipping. Clipping identifies that portion of a material zone which lies inside a mesh element. An example application is shown in Fig. 5; see also [34]. In this example, the TU Berlin logo represents a pseudo-material domain which is clipped against a triangle mesh. The resulting intersection polygons highlighted in different colors correspond to the partial material volumes in each element.

An interface reconstruction procedure will be implemented into the MMALE method developed in this research. Compared to VOF approaches the MOF method is local, that is, it does not require information from neighboring elements or the computation of any terms related to these. Moreover, MOF interface reconstruction works on unstructured meshes and provides an automatic ordering of the materials if an element contains two or more interfaces [2, 23]. The basic equation for both VOF and MOF methods is the volume fraction advection equation

$$
\frac{\partial f^{k}}{\partial t}+\boldsymbol{v} \cdot \nabla f^{k}=0 \quad \text { resp. } \quad \frac{\partial \hat{f}^{k}}{\partial t} \circ \Phi^{-1}+\boldsymbol{c} \cdot \nabla f^{k}=0,
$$



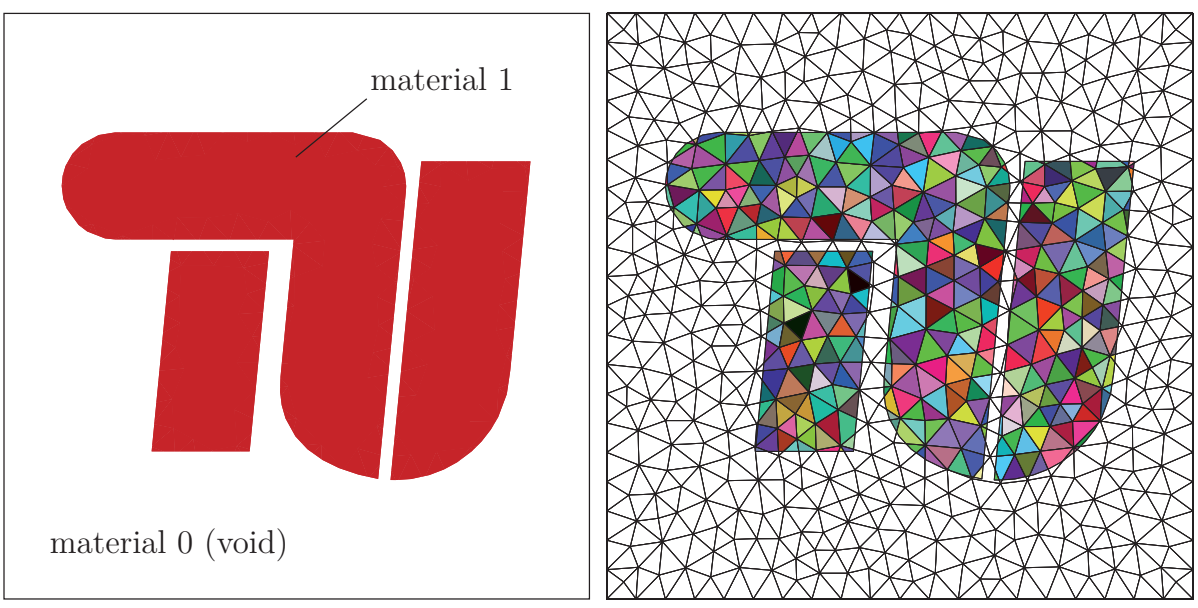

Fig. 5. Clipping of the TU Berlin logo against a triangle mesh. Pseudo-material domains (left) and resulting intersection polygons representing partial material volumes (right); after [34].

with $\hat{f}^{k} \stackrel{\text { def }}{=} f^{k} \circ \Phi$. Finite volume discretization and first-order approximation in time yields a formula similar to (13),

$$
f_{j}^{k+}=\frac{f_{j}^{k-} V_{j}^{-}-\sum_{\mathrm{facets}} \mathrm{Fl}_{k}\left(f^{k}\right) \Delta t}{V_{j}^{+}},
$$

where $\mathrm{Fl}_{k}$ is convective volume flux across a facet of the element boundary which is aware of the spatial distribution of the $k$-th material, i.e. of the interface locations. The material transport volume across a facet is represented by the term $\mathrm{Fl}_{k}\left(f^{k}\right) \Delta t$. After the volume fraction has been updated, the material-associated variable at the end of the remap step can be computed from

$$
q_{j}^{k+}=\frac{f_{j}^{k-} q_{j}^{k-} V_{j}^{-}-\sum_{\text {facets }} \mathrm{Fl}_{k}\left(f^{k} q^{k}\right) \Delta t}{f_{j}^{k+} V_{j}^{+}},
$$

which is a particular form of (13).

\section{$3 \quad$ Experimental Model Tests}

The following section is concerned with the experimental model tests that have been carried out in order to observe the multi-material flow field during vibroinjection pile installation into sand. The main purposes are (i) the verification of the assumptions underlying the theoretical and numerical investigations and (ii) the validation of the MMALE computational models through back-calculation of the model tests. The latter particularly requires a sufficiently detailed description 
of the properties of the test sand in such a way that material constants of the hypoplastic rate constitutive equation can be determined. To the best knowledge of the authors, however, there are no qualified experimental tests reported in the literature that could be used. Therefore, a completely new model test equipment had to be designed and manufactured, and series of tests had to be conducted and analyzed during the course of our research. Preliminary work was done within the scope of student projects and theses at the Chair of Soil Mechanics and Geotechnical Engineering, Technische Universitt Berlin [51, 41, 16, 17].

No attempts have been made to reproduce processes of vibro-injection pile installation in the field. Consequently no scale models or other similitude theoretical issues to achieve field-scale equivalence needed to be considered. Therefore, tests could be conducted at $1 g$ and the use of special test sand could be avoided. The absence of scale effects will also facilitate the validation process without placing any further restrictions on the applicability of the MMALE method. MMALE computational models will be built to back-calculate selected experimental tests "as is", that is, without any scaling.

\subsection{Test Set-up and Measurement Concept}

The test set-up is shown in Fig. 6. Its main components are a waterproof chamber with glass panel serving as a viewing window, a special model pile, and a device for vibratory pile driving. All components are in-house developments. The vibrator consists of two counter-rotating and synchronously revolving imbalances whose mass and rotational speed are adjustable. The dead weight of the vibrator mounted on the pile head is about $100 \mathrm{~kg}$, so that a counter balance becomes necessary in order to keep the penetration velocity of the driven pile acceptable. The model pile is made up of a $50 \mathrm{~mm} \times 50 \mathrm{~mm}$ stainless steel square tube equipped with a welded-on collar at the pile toe as well as a built-in injection tube whose opening is located directly above the collar (Fig. 6c); deflector plates that can be attached to the collar are not shown. The tapering of the toe prevents deviation of the pile from the vertical. Just as with the vibro-injection piles in practice, the shaft annulus created by the collar can be injected with grout material while the pile is being driven into the water-saturated sand. Pressurized injection is enabled by a diaphragm pressure vessel with a maximum operation pressure of 10 bar.

During the tests the pile was guided alongside the glass panel, so that the vibration and grouting process could be digitally filmed through the viewing window of the chamber. A standard Full HD camcorder (Panasonic HDC-SD900) was used to record a series of consecutive still images at $50 \mathrm{~Hz}$ with a maximum resolution of $1920 \times 1080$ pixels. The scene was illuminated by two $500 \mathrm{~W}$ halogen floodlights, which were placed in a large enough distance to the camera in order to minimize heating. Measurement of details of the multi-material flow field without on-sample instrumentation then was enabled by analyzing the recorded image sequence using image correlation software.

In the present research we use GeoPIV [58] for image correlation, a MATLAB toolbox based on particle image velocimetry (PIV). PIV is a two-dimensional 

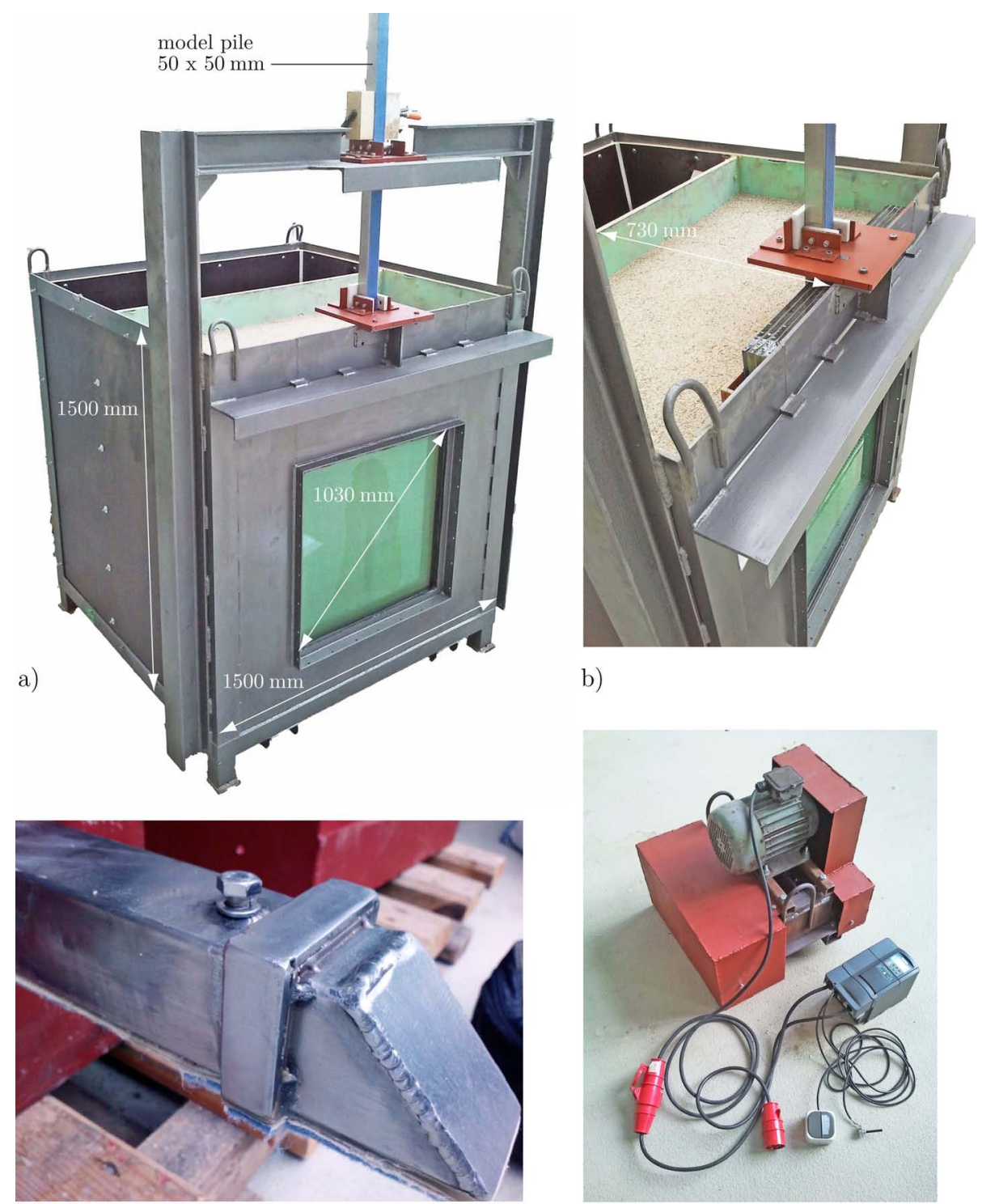

b)

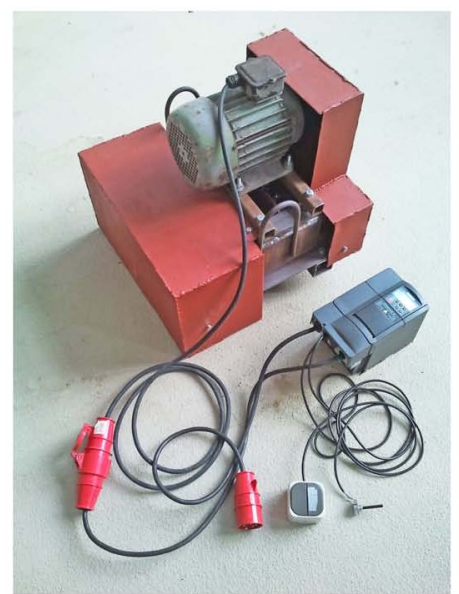

c)

d)

Fig. 6. Experimental investigation of vibro-injection pile installation. a) Filled test chamber with glass panel and model pile. b) Detailed view of the glass panel, pile guide, and model pile. c) Tapered pile toe with welded-on collar and bolt closing the injection tube. d) Self-made vibrator with controller (frequency converter). 
measuring technique for the whole instantaneous spatial velocity field resp. incremental displacement field of a moving and deforming material sample $[1,59,6]$. This is achieved by tracking the texture within areas of an image through a sequence of images. The PIV workflow is as follows. Two images from consecutive configurations of the material sample are subdivided into patches, and then the pixel intensity (luminance) of each pair of patches is cross-correlated in terms of an image displacement vector. The highest correlation occurs when the image displacement vector coincides with the image of the averaged incremental displacement of the material zone captured on the image patch. The totality of local incremental displacement vectors associated with all patches represents the incremental displacement field. From this, the incremental strain field can be obtained through postprocessing.

\subsection{Experimental Program}

The sand employed in the experimental model tests is a quartz sand with wellrounded to angular grains identified as fine-gravelly coarse Sand (fgrCSa) according to [21]. Its grain size distribution curve is plotted in Fig. 7. The limit void ratios are $e_{\min }=0.482$ and $e_{\max }=0.779$, with the void ratio related to the porosity by $e=n /(1-n)$. Further granulometric properties are listed in [6]. In that thesis the same sand was used for quasi-static penetration tests.

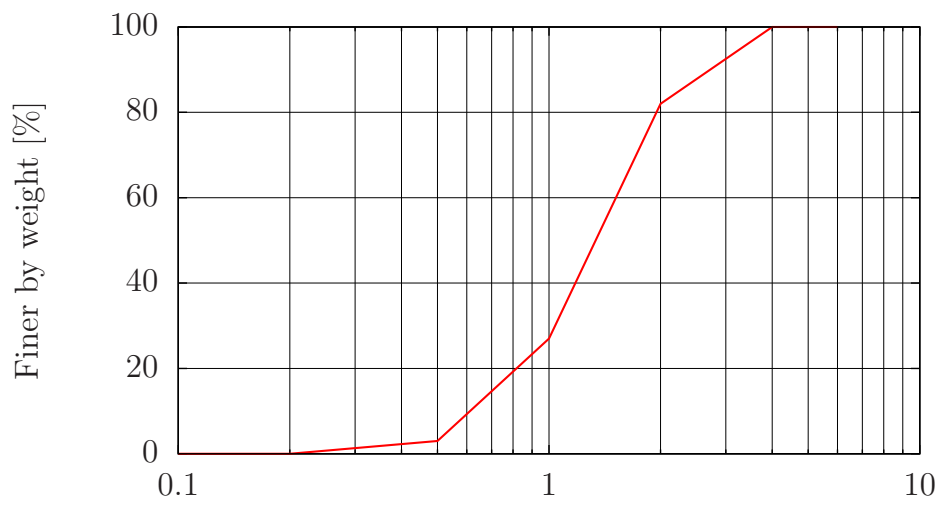

Size of sieve opening $d[\mathrm{~mm}]$

Fig. 7. Grain size distribution curve of the model test sand.

Series with a total of 10 tests have been conducted (Tab. 1). Parameters varied were the counter balance, the degree of saturation, the grouting material, the grouting pressure, and the load amplitude of the vibrator. The vibration frequency was about $20 \mathrm{~Hz}$ for all tests and the load amplitude of the vibrator varied between $1.9 \mathrm{kN}$ and $2.56 \mathrm{kN}$. The static force, that is, the dead weight of 
Table 1. Details of conducted vibro-injection pile (RI-pile) installation tests.

\begin{tabular}{|c|c|c|c|c|}
\hline Test ID & Date & Saturation & $\begin{array}{l}\text { Grouting material / } \\
\text { Pressurization [bar] }\end{array}$ & $\begin{array}{c}\text { Frequency [Hz] / } \\
\text { Load amplitude }[\mathrm{kN}]^{a}\end{array}$ \\
\hline RI-1-D & \multirow{4}{*}{2011} & air dried & \multirow{3}{*}{ none } & $20.7 / 2.56$ \\
\hline RI-2-F & & \multirow{3}{*}{ water flooded } & & $20.0 / 2.39$ \\
\hline $\begin{array}{l}\mathrm{RI}-3-\mathrm{F} \\
\mathrm{RI}-4-\mathrm{F}\end{array}$ & & & & $20.0 / 1.90$ \\
\hline$\overline{\mathrm{RI}-5-\mathrm{B}^{b}}$ & & & bentonite slurry $/-^{c}$ & $20.0 / 2.39$ \\
\hline RI-6-D & \multirow[b]{2}{*}{2012} & air dried & none & \multirow[b]{2}{*}{$20.0 / 2.39$} \\
\hline \begin{tabular}{|l|}
$\mathrm{RI}-7-\mathrm{B}$ \\
$\mathrm{RI}-8-\mathrm{B}$ \\
$\mathrm{RI}-9-\mathrm{H}^{b}$ \\
\end{tabular} & & water flooded & $\begin{array}{l}\text { bentonite slurry / }-^{c} \\
\text { bentonite slurry / } 1.0 \\
\text { hydraulic binder / 3.0 }\end{array}$ & \\
\hline
\end{tabular}

$\mathrm{D}$ - dry; F - flooded; B - bentonite; $\mathrm{H}$ - hydraulic binder

In all tests the initial mean relative density was $>85 \%$ (very dense).

${ }^{a}$ with respect to the vibrator; the static force (dead weight) varies between the tests

${ }^{b}$ analyzed by using particle image velocimetry

${ }^{c}$ loaded under its own weight

the vibrator and the pile was varied between the tests by using different counter balances.

In all tests the chamber was filled with air dried sand by dry sieve pluviation. Each sand model was prepared in several layers of equal thickness in order to achieve a homogeneous distribution of initial density. The minimum height of pluviation for the first layer of sand is restricted by the inner height of the chamber such that the initial mean relative density was always larger than $85 \%$ (very dense). Two tests were run in air dried sand, whereas the other were carried out in sand which had been water flooded.

In three tests the pile shaft annulus created by the welded-on collar was injected with pigmented bentonite slurry. In two other experiments we tested a hydraulic binder commonly used in geotechnical engineering for filling, sealing, and solidification.

\subsection{Preliminary Test Results}

Figure 8 shows a digital photograph of test RI-8-B through the viewing window of the chamber right after the installation of the pile has completed. It can be seen from the figure that the bentonite slurry infiltrated into the pore space of the test sand although the mass concentration of bentonite in the slurry is pretty high. It fails in keeping open the shaft annulus. In contrast to that, the hydraulic binder has a sufficiently high shear strength to stabilize the shaft annulus while possessing excellent flowability during pressurized grouting. Concerning test RI9-H shown in Fig. 9, this is indicated by the clear soil-grout interface which is almost vertical along the pile shaft. Infiltration of the coarse test sand cannot 
be completely avoided, that is to say, the model assumption of an impermeable soil-grout interfaces does not appear as a reasonable one. However, it should be noted that the amount of infiltrated hydraulic binder increased with time and that the still images in Fig. 9 were recorded about half an hour after the pile installation has completed.

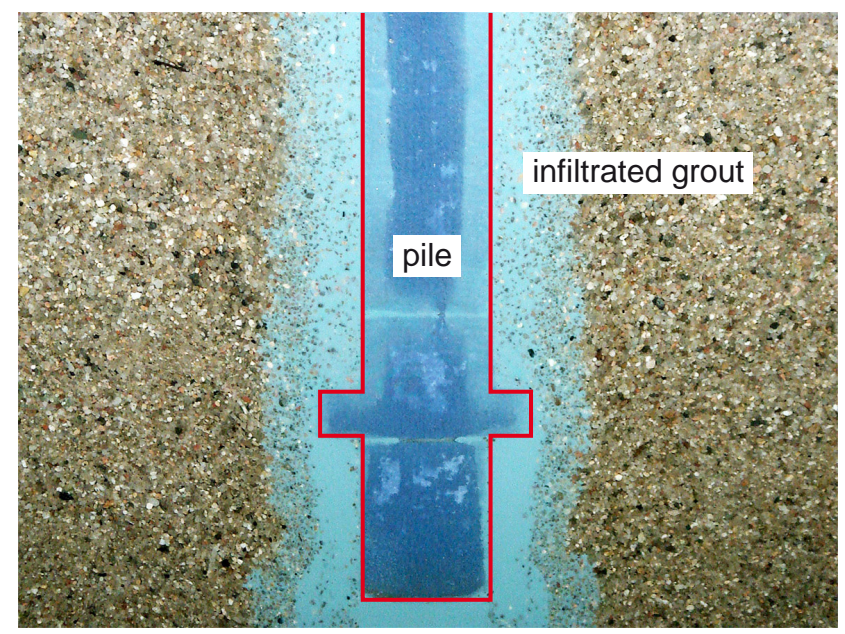

Fig. 8. Digital photograph of the configuration of test RI-8-B (bentonite slurry injection) through the viewing window of the chamber right after pile installation has completed.

The results of a PIV analysis of test RI-5-B using bentonite slurry injection is displayed in Fig. 10. Figure 10a shows the time history of the vertical displacement of the pile tip. Those configurations where image capturing took place are marked with black squares. Concerning a time-averaged motion the pile continuously penetrates the soil due to its self-weight. During a vibration cycle, however, the pile moves upward and downward. The displacement increments in the soil which occurred during the downward motion of the pile between image 1 and image 2 are displayed in Fig. 10b using arrows with scaled length. It is clearly visible that the soil is not only displaced below the pile toe and underneath the collar in a predominantly vertical direction but also moves downward above the collar. Figure 10c shows the displacement increments due to upward motion of the pile between image 5 and image 6 . Qualitatively the same soil motion can be observed as in Fig. 10b but with reverse signs, indicating that the soil located at the pile shaft is dragged along with the pile motion. The heavings beneath the pile toe and the collar result from the release of the previously compressed soil. 

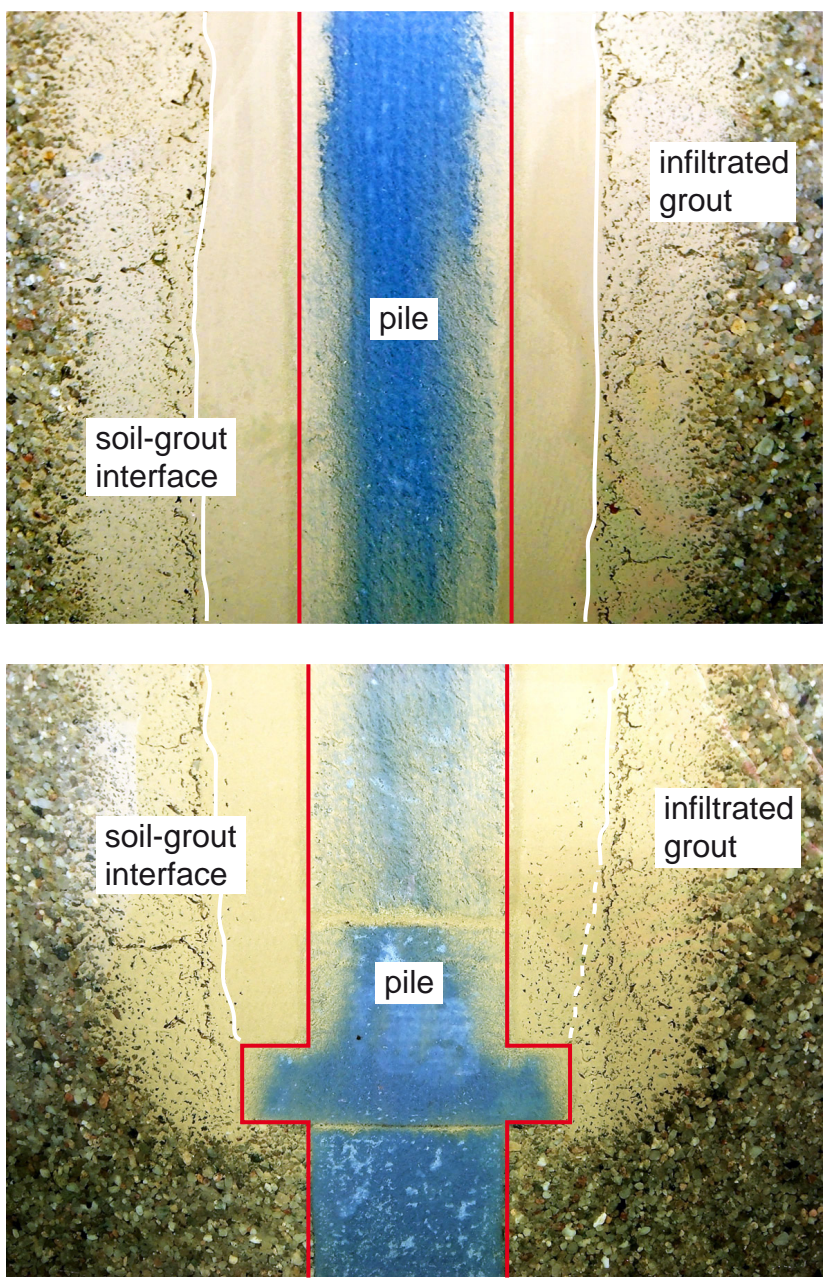

Fig. 9. Digital photograph of the configuration of test RI-9-H (hydraulic binder injection) through the viewing window of the chamber about half an hour after pile installation has completed. 


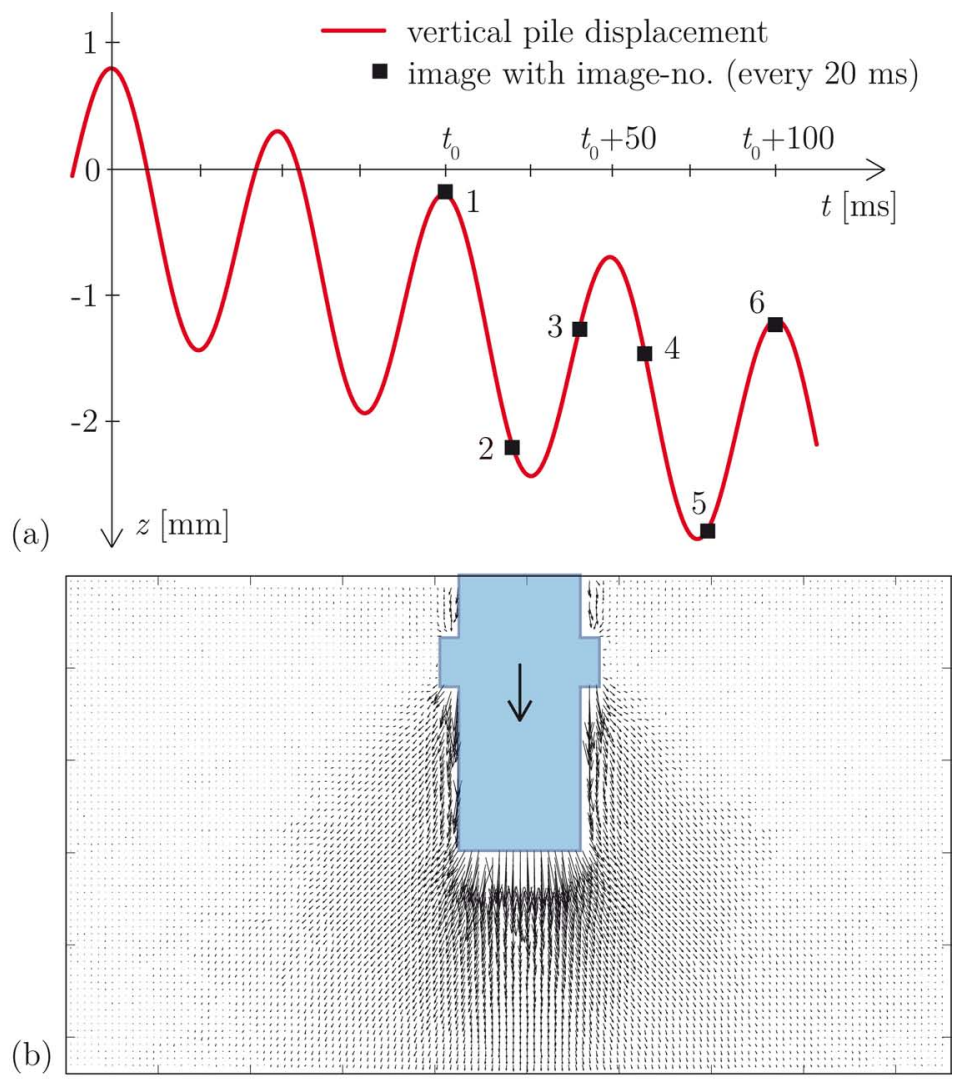

(b)

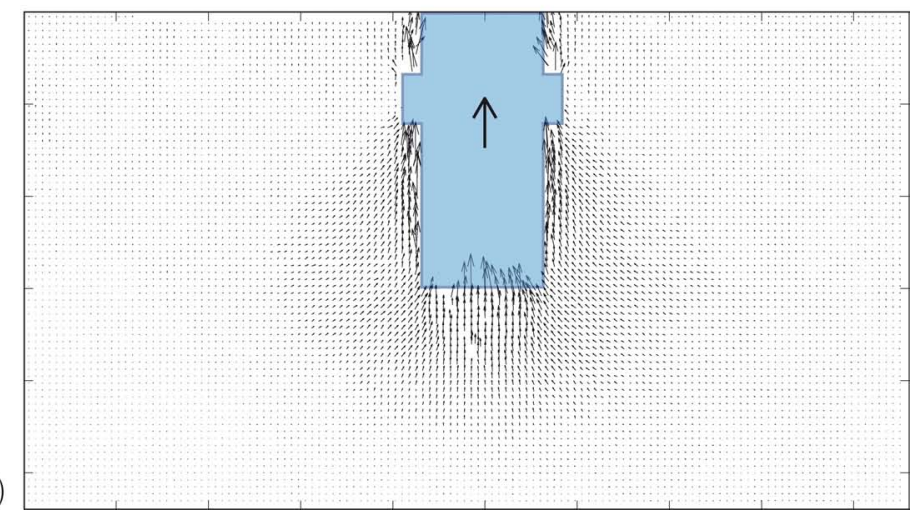

Fig. 10. Results of model test RI-5-B using bentonite slurry injection. (a) Schematic time history of vertical pile displacements. PIV results showing soil displacement increments (b) at upward motion of the pile (image 1 to image 2), and (c) at downward motion of the pile (image 5 to image 6). 


\section{Numerical Examples and Validation}

The following section presents some numerical examples which belong to a verification and validation $(\mathrm{V} \& \mathrm{~V})$ process controlling and ensuring quality of our new MMALE method. Particular algorithms of the method, like the transport algorithm in the remap step, are verified by running patch tests and basic initial boundary value problems. The validation of the complete MMALE method is enabled through back-analysis of data resulting from the experimental model tests carried out or from example problems found in the literature. Since the numerical implementation of the multi-material option of the method (interface reconstruction, etc.) is still in progress, all the examples shown here consider only a single material per element and time step. Therefore, the computational models used do not utilize all features of MMALE but have to be considered as simplified ALE models.

\subsection{Verification of the Transport Algorithm}

Example problems towards verification of the advective transport algorithm use a fixed (Eulerian) mesh and prescribe a steady velocity field of the material in conjunction with an initial distribution of a scalar field. The scalar field has no specific physical meaning but represents the quantity being advected through the mesh. In the present case it can be associated with the solution obtained at the end of the Lagrangian step, e.g. a stress component.

Figure 11a shows the almost uniform unstructured mesh used in the example. The initial signal (Fig. 11b) is a cylindrical pulse implemented as follows. The color function is set to zero in all elements except for those in the circular zone highlighted in Fig. 11a, where the color function is set to 100. For visualization the element-centered values are copied to the nodes and then averaged. In the next step, a steady velocity field is prescribed in such a way that the material performs a full 360 clockwise rigid rotation about the center of the square domain in 720 advection steps. This means that the analytical solution at the final state and the initial cylindrical pulse are identical. The mesh is kept fixed, meaning that the MMALE method is run in the purely Eulerian mode.

The results of the implemented first-order transport algorithm after one half rotation and after a full rotation are plotted in Figs. 11c and 11d, respectively. The dashed circle represents the analytical solution. The gradual increase of the area were the color function has values greater than zero indicates numerical diffusion introduced by the finite volume approximation. The plateau erodes and the steep gradients present in the initial signal are getting smeared during the course of rotation. Moreover, the peak moves radially inward, and its maximum value after a full rotation is reduced to only $57 \%$ of its initial value. As already mentioned, numerical diffusion would be less pronounced if a secondor higher-order advective transport algorithm would be used. However, we note that rotation of a cylindrical pulse constitutes an academic extreme example as the solution variables in practical soil mechanical problems often have smaller gradients. Moreover, in the operator-split MMALE method, every transport step 


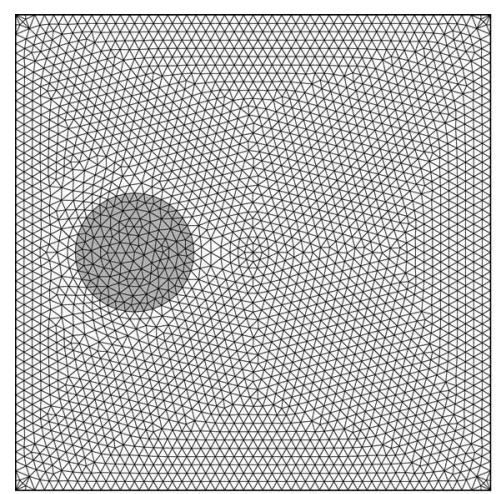

(a) mesh

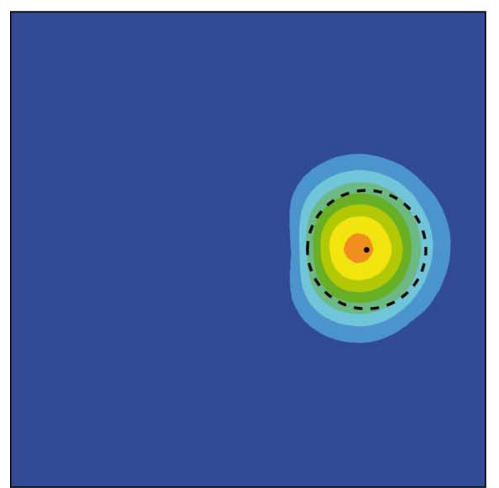

(c) after one half rotation

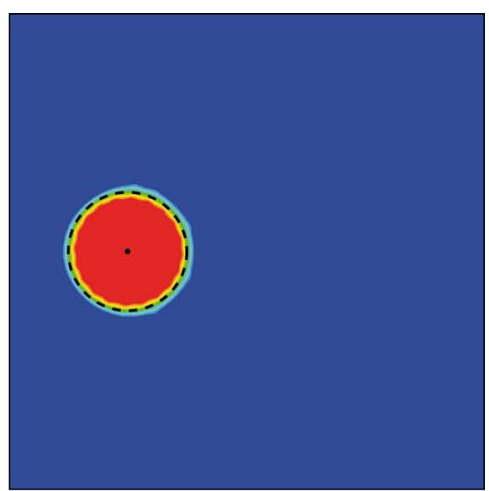

(b) initial scalar distribution

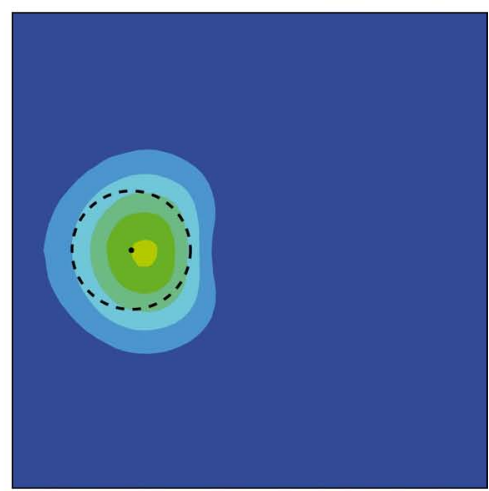

(d) after a full rotation

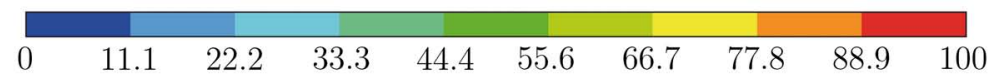

Fig. 11. Rotational advection of a cylindrical pulse by the implemented first-order transport algorithm. The analytical solution is indicated by the dashed circle. 
is followed by a Lagrangian step that should bring back the solution variables to an admissible state.

\subsection{Piercing Test}

Piercing is test problem borrowed from the metal forming community which can be employed to validate the MMALE method. In this quasi-static process a billet is held in a heavy walled container and hollowed out by a flat punch (Fig. 12). The example is a plane strain problem and assumes the punch to be rigid and perfectly rough. The vertical and horizontal walls of the container are smooth to ensure sliding contact conditions in the container-billet interface. For the case of plastic-rigid material and a container to punch breadth ratio of $S / B=0.5$, the maximum penetration pressure, $p$, in a steady piercing process is related to yield stress in uniaxial tension through $p / \sigma^{\mathrm{y}}=(2+\pi) / \sqrt{3}[28]$. This is the same relation as for the ultimate bearing capacity of a strip footing on the plane surface of a weightless and frictionless cohesive soil.

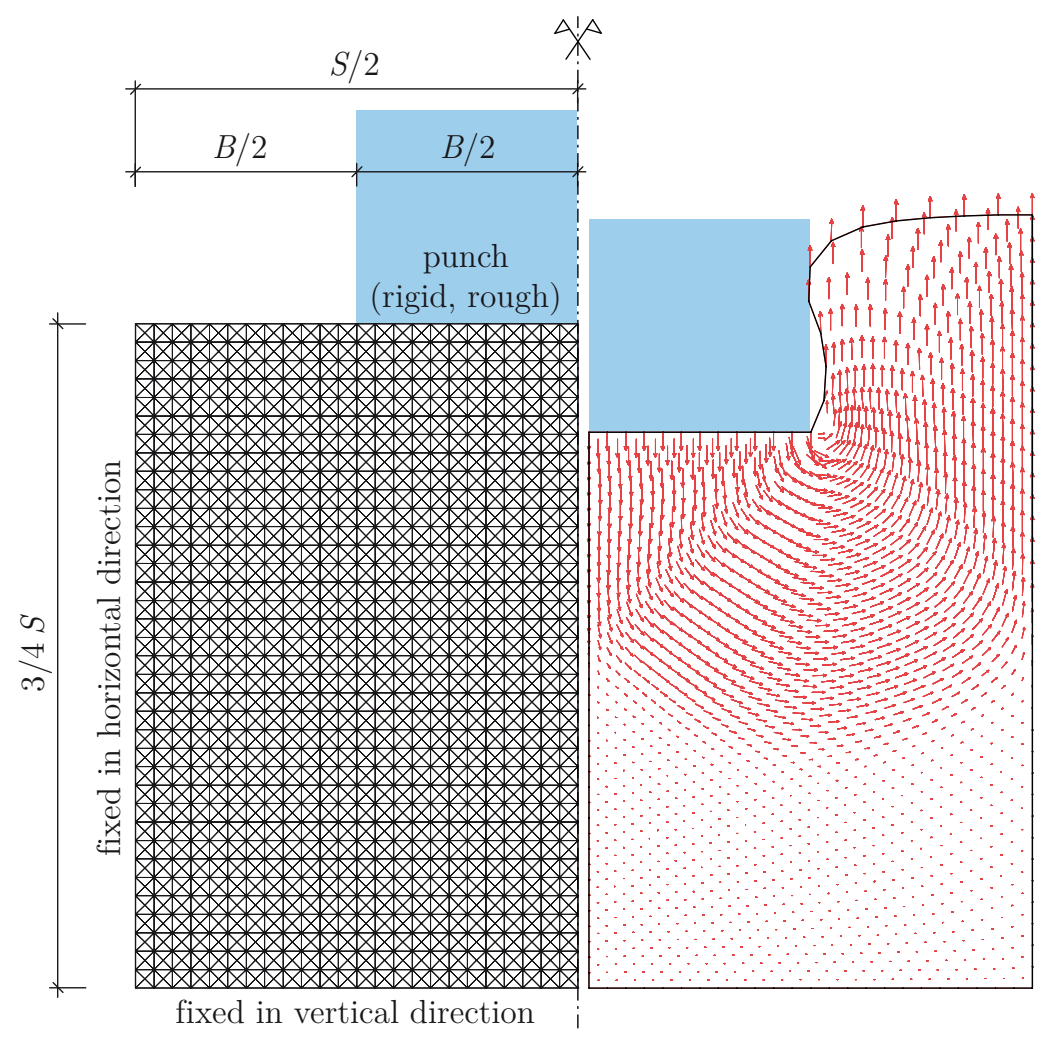

Fig. 12. Simulation of a piercing process. Problem statement and coarse structured mesh (left), deformed domain and velocity field at $z / B=0.25$ (right). 
The two finite element models used for back-calculation of the analytical solution take advantage of symmetry and differ only in the coarseness of the mesh. The model and the coarse mesh are shown in Fig. 12 left. No contact elements were used. The plastic-rigid material of the billet is modeled by a hypoelasto- $J_{2}$-plastic rate constitutive equation (von Mises plasticity). The set of material constants chosen for the simulations consists of Young's modulus $E=2600 \mathrm{kPa}$, Poisson's ratio $\nu=0.3$, initial yield stress $\sigma^{\mathrm{y} 0}=20 \mathrm{kPa}$, and plastic modulus $E^{\mathrm{p}}=0 \mathrm{kPa}$ (ideal plastic response).

Figure 12 on the right plots the deformed computational domain at a relative penetration depth of $z / B=0.25$ together with the velocity field on the billet obtained with the coarse mesh. The sudden change in flow direction below the punch is related to a slip line that intersects the axis of symmetry at $45^{\circ}$. A second, less apparent slip line intersects the wall of the container at $45^{\circ}$. The unrealistic deformation of the unconstrained material boundary lateral to the punch (Fig. 12 right) is partly due to its simplified treatment in the ALE method without the multi-material option, but the problem could be resolved in this example by using a finer mesh.

The calculated load-displacement curves and the analytical solution are plotted in Fig. 13. In contrast to the plastic-rigid material behavior assumed in [28], the rate equation governing hypoelastic response in the ALE simulation results in a gradual increase of the punch pressure along with increasing indentation. The relative pressure $p / \sigma^{\mathrm{y}}=2.97$ of the analytical solution is, however, reached at relative penetration depths of less than $z / B=0.05$ in all calculations. Beyond that point, the simulated pressure is always larger than the plastic-rigid solution, but the difference decreases with decreasing element size.

\subsection{Pile Penetration into Sand}

Until today the realistic simulation of pile penetration into sand is one of the most challenging problems in soil mechanics because of the complex material behavior, large deformations, and contact constraints. Stable and robust simulations are hard to achieve, even when ALE methods are employed. Loading has to be increased very slowly, and adjusting the contact parameters at the beginning of the simulation only is a science of its own.

Recent numerical results of a succeeded ALE simulation of quasi-static pile penetration into sand are shown in Fig. 14. The pile is assumed smooth and rigid, and the initial void ratio of the sand was chosen to $e_{0}=0.678$ (initial relative density $\left.D_{\mathrm{r} 0}=0.34\right)$. The initial configuration has a very simple geometry because the penetration was started at the ground surface. Contact elements are attached to the pile and soil surfaces, and a Lagrange multiplier contact algorithm enforces zero penetration of the pile elements when contact is closed. The number of axisymmetric solid elements used for the simulation is approximately 36000 , with the centerline of the pile serving as the axis of radial symmetry. All nodes at the lower boundary of the mesh are fixed in vertical direction, and the nodes of both vertical boundaries are fixed in radial direction. initial The initial stress state within the sand was prescribed as a $K_{0}$-state. 


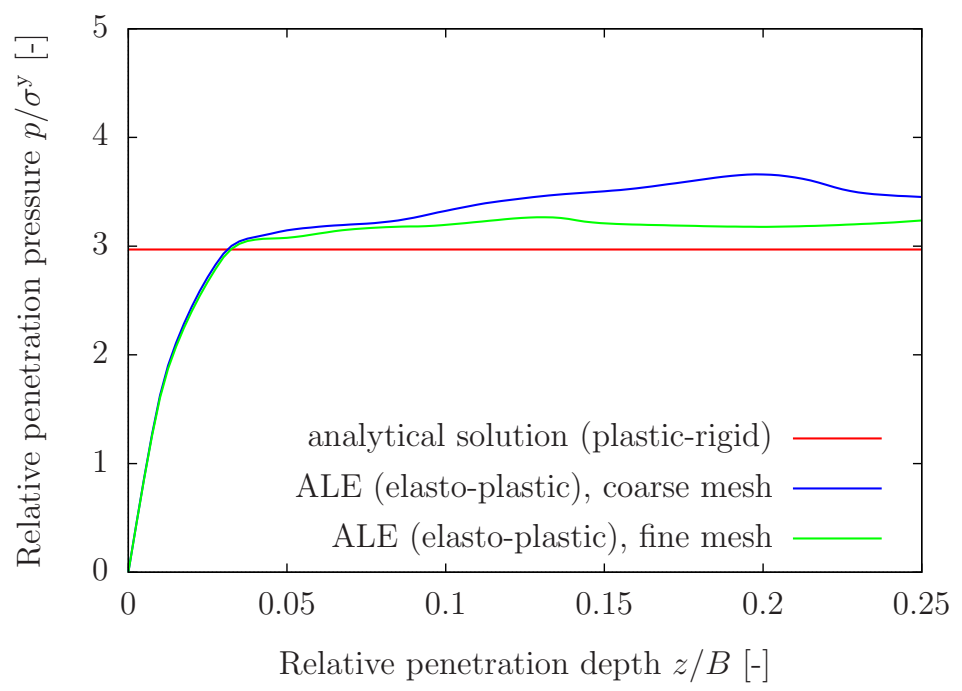

Fig. 13. Simulation of a piercing process. Analytical solution $p / \sigma^{\mathrm{y}}=(2+\pi) / \sqrt{3}$ for plastic-rigid material [28] and results of an ALE simulation using the hypoelasto- $J_{2}$ plastic model (von Mises plasticity).

Figure 14 plots the deformed configuration and the predicted void ratio distribution at a relative penetration depth of $z / D=5.0$, where $D$ is the pile diameter. Recall that void ratio is a material state variable in the formulation of the hypoplastic rate constitutive equation used to model the mechanical behavior of sand. Hence, its spatial distribution is generally affected by advection during the ALE remap step. The figure indicates a significant densification of the sand immediately underneath the pile base at that stage of penetration. The hemispherical shape and the smooth skin of the pile prevents soil from being trapped in a core zone ahead of the pile base. The sand continuously expands through shearing once the pile base has passed. Consequently, the pile shaft is surrounded by a loosening zone. Since the pile displaces the soil, soil heaving occurs lateral to the pile.

As shown in Fig. 15, the predicted load-displacement curve of the quasi-static pile penetration is in good agreement with experiments [6]. However, the curves considerably differ in relative penetration depths of $z / D<1$ and $z / D>5$. The difference at small penetration depths is related to the undesired restraining forces in the experimental set-up not accounted for in the numerical model as well as to the problem of modeling the behavior of sand at very low effective stress levels. In larger depths, the predicted load-displacement curves show a characteristic zig-zag form which stems from the contact interface approximation [53]. 


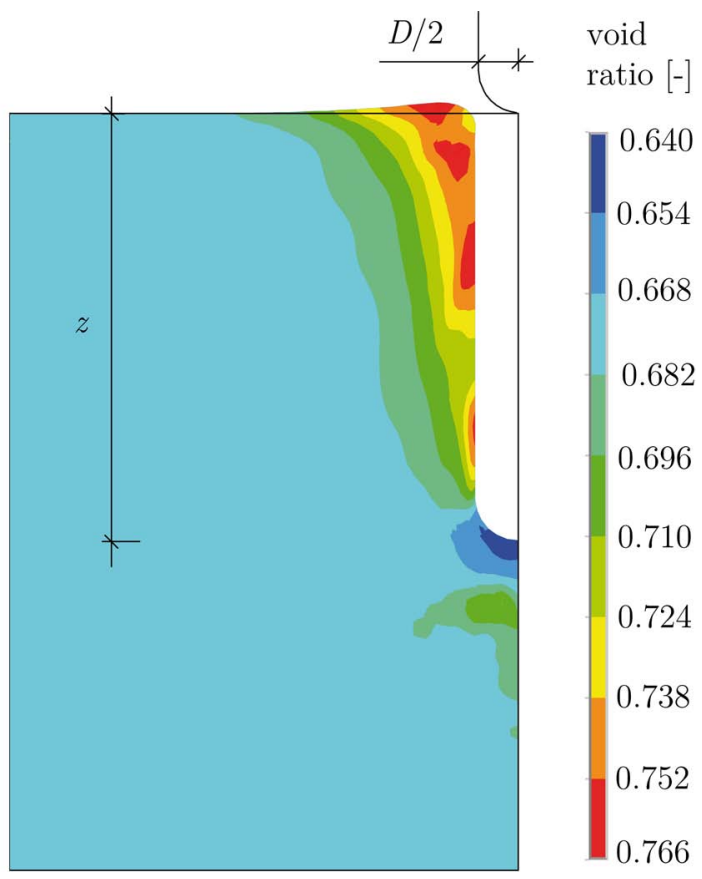

Fig. 14. Quasi-static penetration of a smooth rigid pile into medium dense sand (initial void ratio $e_{0}=0.678$ resp. initial relative density $D_{\mathrm{r} 0}=0.34$ ). Edges of the undeformed configuration, deformed configuration, and predicted void ratio distribution at a relative penetration depth of $z / D=5.0$. 


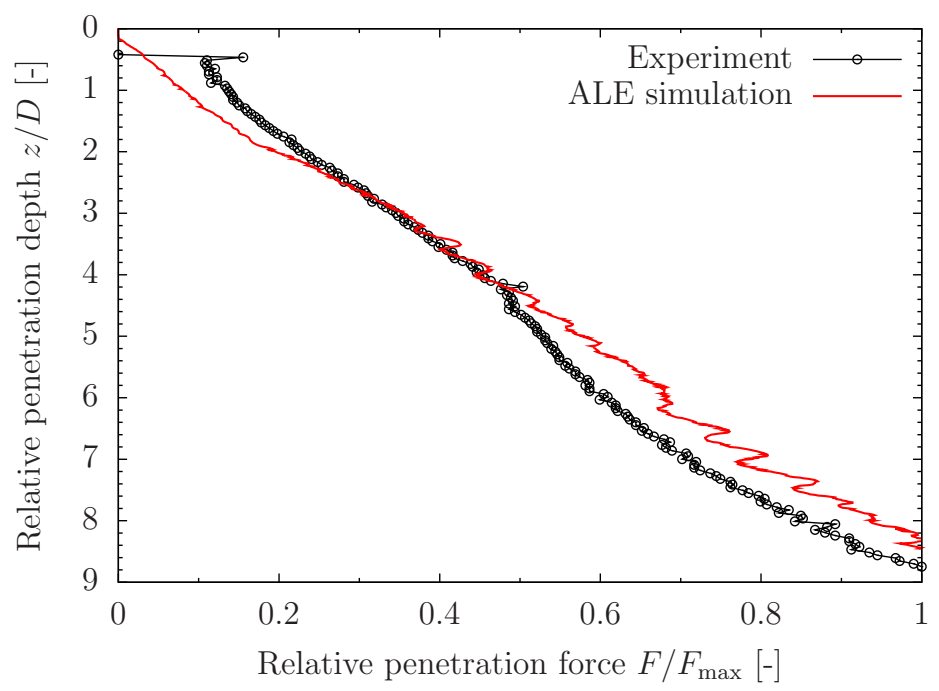

Fig. 15. Quasi-static penetration of a smooth rigid pile into medium dense sand (initial void ratio $e_{0}=0.678$ resp. initial relative density $D_{\mathrm{r} 0}=0.34$ ). Comparison of measured and predicted load-displacement curves.

\section{Conclusions and Outlook}

We have developed a multi-material arbitrary Lagrangian-Eulerian (MMALE) method to numerically model and simulate vibro-injection pile installation in water-saturated sand. Because of its applicability on low-velocity problems related to porous media formed by granular material the method is unique on national as well on international level and offers great potential for future research. MMALE describes the motion of the computational mesh independent of the material motion. Material interfaces can flow through the mesh, so that mesh elements may arise which contain two or more materials. Concerning these multi-material elements the two-equation reduced model derived in Part 1 of these papers is applied in order to relate in a mechanically consistent way the element-associated variables with those of the multi-material flow.

The numerical implementation of the method is based on a Lagrange-remap strategy through which each calculational cycle is divided into a Lagrangian step and a remap step. This allows for simpler and more robust algorithms compared to the monolithic problem as well as the implementation into Lagrangian finite element programs. As the remap step contributes most to the overall cost of the calculation, several Lagrangian steps can be performed before the total transport associated with those steps is accounted for in a single remap step.

The Lagrangian step is largely identical to the standard step for advancing solution in time in implicit Lagrangian finite element programs. The mesh follows the material as it deforms, and the rate constitutive equations of the 
particular materials are integrated over the time step by taking into account the requirement of incremental objectivity. In order to realistically model the behavior of water-saturated sand, especially the tendency to liquefy under cyclic loads, the MMALE method is combined with a highly-developed hypoplastic rate constitutive equation advanced in the Subproject 1 of this DFG Research Unit. Furthermore, in multi-material elements the evolution of the volume fractions must be taken into account because every constituent is generally compressible.

In the remap step the nodes of the element mesh are either moved back to their original positions (Eulerian mode) or relocated such that the overall mesh quality is kept in an acceptable range (ALE mode). The relocation of nodes must be supplemented by a numerical transport of material resp. solution variables relative to the mesh, so as to achieve mechanically consistency. This is implemented by using first-order linear and high-resolution non-linear conservative transport algorithms from the field of computational fluid dynamics. The simple first-order transport algorithm of the Godunov-type currently implemented is, however, sufficient for many applications. In order to precisely determine the amount of lost or gained material volume during the remap step in multi-material elements, interfaces are reconstructed and tracked along with the flow field by using VOF resp. MOF methods.

Parallel to the theoretical and numerical investigations, experimental model tests concerning the installation of vibro-injection piles in sand have been carried out in order to investigate the relevant installation phenomena and to provide data that can be back-calculated using the MMALE method. A test chamber with viewing window together with a special model pile have been designed and manufactured for this purpose. The model pile, like the steel profiles used for vibro-injection piles in practice, allows for the pressurized injection of the pile shaft above a welded-on collar located at the pile toe. During the tests installation process has been digitally filmed through the viewing window, and then the recorded image sequence has been analyzed by using particle image velocimetry. The tests reveal that the grains in a body of sand usually entail adequate image texture for PIV analysis, but not the grouting material unless it would be seeded with marker particles.

The research work reported here is still in progress. Open issues include the completion of the MMALE implementation, the development of MMALE computational models for the simulation of grouting and vibro-injection pile installation in sand, as well as the validation of the computational models through back-calculation of selected experimental model tests.

Acknowledgments. The presented work was carried out under the financial support from the German Research Foundation (DFG; grants SA 310/26-1 and SA 310/26-2) as part of the DFG Research Unit FOR 1136, which is gratefully acknowledged. We thank our colleagues in this research unit for several fruitful discussions about our work. 


\section{References}

1. R. J. Adrian. Particle-Imaging Techniques for Experimental Fluid Mechanics. Annual Review of Fluid Mechanics, 23:261-304, 1991.

2. H. T. Ahn and M. Shashkov. Multi-Material Interface Reconstruction on Generalized Polyhedral Meshes. Journal of Computational Physics, 226:2096-2132, 2007.

3. D. N. Arnold, F. Brezzi, and M. Fortin. A Stable Finite Element for the Stokes Equations. Calcolo, 21(4):337-344, 1984.

4. H. Askes, L. Bodé, and L. J. Sluys. ALE Analysis of Localization in Wave Propagation Problems. Mechanics of Cohesive-Frictional Materials, 3:105-125, 1998.

5. D. Aubram. Differential Geometry Applied to Continuum Mechanics. Number 44 in Veröffentlichungen des Grundbauinstitutes der Technischen Universität Berlin. Shaker Verlag, Aachen, 2009.

6. D. Aubram. An Arbitrary Lagrangian-Eulerian Method for Penetration into Sand at Finite Deformation. Number 62 in Veröffentlichungen des Grundbauinstitutes der Technischen Universität Berlin. Shaker Verlag, Aachen, 2013.

7. D. Aubram. Optimization-Based Smoothing Algorithm for Triangle Meshes over Arbitrarily Shaped Domains. Finite Elements in Analysis and Design, submitted for publication.

8. D. Aubram, F. Rackwitz, and S. A. Savidis. An ALE Finite Element Method for Cohesionless Soil at Large Strains: Computational Aspects and Applications. In T. Benz and S. Nordal (eds.): Proceedings 7th European Conference on Numerical Methods in Geotechnical Engineering (NUMGE), pages 245-250. CRC Press, Boca Raton, 2010.

9. T. Barth and M. Ohlberger. Finite Volume Methods: Foundation and Analysis. In E. Stein, R. de Borst, and T. J. R. Hughes (eds.): Encyclopedia of Computational Mechanics, vol. 1, chapter 15. John Wiley \& Sons, Ltd., 2004.

10. T. Belytschko, W. K. Liu, and D. Moran. Nonlinear Finite Elements for Continua and Structures. John Wiley \& Sons, Ltd., 2000.

11. D. J. Benson. Computational Methods in Lagrangian and Eulerian Hydrocodes. Computer Methods in Applied Mechanics and Engineering, 99:235-394, 1992.

12. D. J. Benson. A Multi-Material Eulerian Formulation for the Efficient Solution of Impact and Penetration Problems. Computational Mechanics, 15:558-571, 1995.

13. D. J. Benson. A Mixture Theory for Contact in Multi-Material Eulerian Formulations. Computer Methods in Applied Mechanics and Engineering, 140:59-86, 1997.

14. D. J. Benson. An Implicit Multi-Material Eulerian Formulation. International Journal for Numerical Methods in Engineering, 48:475-499, 2000.

15. D. J. Benson. Volume of Fluid Interface Reconstruction Methods for MultiMaterial Problems. Applied Mechanics Reviews, 55(2):151-165, 2002.

16. O. Beyer. Erweiterung und Optimierung eines Modellversuchsstandes fr die Simulation der Rüttelinjektionspfahlherstellung. Bachelor's Thesis, Technische Universität Berlin, Germany, 2012 (unpublished; in German).

17. M. Büchler. Qualitative Untersuchung bodenmechanischer Phänomene whrend der Herstellung von Rüttelinjektionspfählen mittels Particle Image Velocimetry. Bachelor's Thesis, Technische Universität Berlin, Germany, 2013 (unpublished; in German).

18. Ch. Carow. Das MINI-Element fr Fluid-Kornstruktur-gekoppelte Problemstellungen. Master's Thesis, Technische Universität Berlin, Germany, 2012 (unpublished; in German).

19. P. Colella, H. M. Glaz, and R. E. Ferguson. Multifluid Algorithms for Eulerian Finite Difference Methods. 1997 (unpublished manuscript). 
20. R. B. DeBar. Fundamentals of the KRAKEN Code. Technical Report UCID-17366, Lawrence Livermore Laboratory, Livermore, USA, 1974.

21. DIN EN ISO 14688-1. Geotechnische Erkundung und Untersuchung — Benennung, Beschreibung und Klassifizierung von Boden - Teil 1: Benennung und Beschreibung. Beuth Verlag, Berlin, January 2003 (German Code).

22. V. Dyadechko and M. Shashkov. Moment-of-Fluid Interface Reconstruction. Technical Report LA-UR-05-7571, Los Alamos National Laboratory, New Mexico, USA, 2005.

23. V. Dyadechko and M. Shashkov. Reconstruction of Multi-Material Interfaces From Moment Data. Journal of Computational Physics, 227:5361-5384, 2008.

24. D. Freßmann and P. Wriggers. Advection Approaches for Single-and MultiMaterial Arbitrary Lagrangian-Eulerian Finite Element Procedures. Computational Mechanics, 39:153-190, 2007.

25. J. Glimm, E. Isaacson, D. Marchesin, and O. McBryan. Front Tracking for Hyperbolic Systems. Computational Mechanics, 2:91-119, 1981.

26. J. Glimm, J. W. Grove, X. L. Li, K.-M. Shyue, Y. Zeng, and Q. Zhang. ThreeDimensional Front Tracking. SIAM Journal on Scientific Computing, 19(3):703-727, 1998.

27. A. Harten. High Resolution Schemes for Hyperbolic Conservation Laws. Journal of Computational Physics, 49(3):357-393, 1983.

28. R. Hill. The Mathematical Theory of Plasticity. Oxford University Press, 1998 (Reprint; first published 1950).

29. C. W. Hirt and B. D. Nichols. Volume of Fluid (VOF) Method for the Dynamics of Free Boundaries. Journal of Computational Physics, 39:201-225, 1981.

30. C. W. Hirt, A. A. Amsden, and J. L. Cook. An Arbitrary Lagrangian-Eulerian Computing Method for all Flow Speeds. Journal of Computational Physics, 14:227253, 1974.

31. T. J. R. Hughes and J. Winget. Finite Rotation Effects in Numerical Integration of Rate Constitutive Equations Arising in Large-Deformation Analysis. International Journal for Numerical Methods in Engineering, 15(12):1862-1867, 1980.

32. T. J. R. Hughes. Numerical Implementation of Constitutive Models: RateIndependent Deviatoric Plasticity. In S. Nemat-Nasser, R. J. Asaro, and G. A. Hegemier (eds.): Theoretical Foundation for Large-Scale Computations for Nonlinear Material Behavior, pages 29-63. Martinus Nijhoff Publishers, Dordrecht, Niederlande, 1984.

33. J. M. Hyman. Numerical Methods for Tracking Interfaces. Physica D 12:396-407, 1984.

34. J. Kirchner. Bestimmung der Schnittpolygone fr die Interface-Rekonstruktion in unstrukturierten Dreiecksnetzen. Seminar Paper, Technische Universität Berlin, Germany, 2014 (unpublished; in German).

35. R. J. LeVeque. Finite Volume Methods for Hyperbolic Problems. Cambridge University Press, Cambridge, UK, 3rd ed., 2002.

36. R. W. Lewis and B. A. Schrefler. The Finite Element Method in the Static and Dynamic Deformation and Consolidation of Porous Media. John Wiley \& Sons, Chichester, 2nd ed., 1998.

37. G. Luttwak and R. L. Rabie. The Multi Material Arbitrary Lagrangian Eulerian Code MMALE and Its Application to Some Problems of Penetration and Impact. Technical Report LA-UR-85-2311, Los Alamos National Laboratory, Los Alamos, New Mexico, USA, 1985.

38. J. M. McGlaun and S. L. Thompson. CTH: A Three-Dimensional Shock Wave Physics Code. International Journal of Impact Engineering, 10:351-360, 1990. 
39. G. H. Miller and E. G. Puckett. A High-Order Godunov Method for Multiple Condensed Phases. Journal of Computational Physics, 128:134-164, 1996.

40. A. Niemunis and I. Herle. Hypoplastic Model for Cohesionless Soils with Elastic Strain Range. Mechanics of Cohesive-Frictional Materials, 2:279-299, 1997.

41. D. Noack. Anwendung der digitalen Bildkorrelation auf Modellversuche zur Herstellung von Rüttelinjektionspfählen. Diploma Thesis, Technische Universität Berlin, Germany, 2011 (unpublished; in German).

42. S. Osher and J. A. Sethian. Fronts Propagating with Curvature-Dependent Speed: Algorithms Based On Hamilton-Jacobi Formulations. Journal of Computational Physics, 79:12-49, 1988.

43. S. Osher and R. P. Fedkiw. Level Set Methods: An Overview and Some Recent Results. Journal of Computational Physics, 169:463-502, 2001.

44. J. S. Peery and D. E. Carroll. Multi-Material ALE Methods in Unstructured Grids. Computer Methods in Applied Mechanics and Engineering, 187:591-619, 2000.

45. J. E. Pilliod and E. G. Puckett. Second-Order Accurate Volume-of-Fluid Algorithms for Tracking Material Interfaces. Journal of Computational Physics, 199:465502, 2004.

46. W. J. Rider and D. B. Kothe. Reconstructing Volume Tracking. Journal of Computational Physics, 141:112-152, 1998.

47. A. Rodríguez-Ferran, F. Casadei, and A. Huerta. ALE Stress Update for Transient and Quasistatic Processes. International Journal for Numerical Methods in Engineering, 43:241-262, 1998.

48. M. Rudman. Volume-Tracking Methods for Interfacial Flow Calculations. International Journal for Numerical Methods in Fluids, 24(7):671-691, 1997.

49. S. A. Savidis, D. Aubram, and F. Rackwitz. Arbitrary Lagrangian-Eulerian Finite Element Formulation for Geotechnical Construction Processes. Journal of Theoretical and Applied Mechanics, 38(1-2):165-194, 2008.

50. R. Scardovelli and S. Zaleski. Direct Numerical Simulation of Free-Surface and Interfacial Flow. Annual Review of Fluid Mechanics, 31:567-603, 1999.

51. T. Schulz. Konzeption und Realisierung eines kleinmaßstäblichen Modellversuchsstandes fr die Herstellung von Rüttelinjektionspfählen. Diploma Thesis, Technische Universität Berlin, Germany, 2011 (unpublished; in German).

52. J. A. Sethian. Level Set Methods: Evolving Interfaces in Geometry, Fluid Mechanics, Computer Vision and Material Science. Cambridge University Press, 1996.

53. J. C. Simo and G. Meschke. A New Class of Algorithms for Classical Plasticity Extended to Finite Strains. Application to Geomaterials. Computational Mechanics 11:253-278, 1993.

54. C. Truesdell and W. Noll. In S. S. Antman (ed.): The Non-Linear Field Theories of Mechanics. Springer-Verlag Berlin Heidelberg New York, 3rd ed., 2004.

55. G. Tryggvason, B. Bunner, A. Esmaeeli, D. Juric, N. Al-Rawahi, W. Tauber, J. Han, S. Nas, and Y.-J. Janz. A Front-Tracking Method for the Computations of Multiphase Flow. Journal of Computational Physics, 169:708-759, 2001.

56. E. Vitali and D. J. Benson. An Extended Finite Element Formulation for Contact in Multi-Material Arbitrary Lagrangian-Eulerian Calculations. International Journal for Numerical Methods in Engineering, 67:1420-1444, 2006.

57. P.-A. von Wolffersdorff. A Hypoplastic Relation for Granular Materials with a Predefined Limit State Surface. Mechanics of Cohesive-Frictional Materials, 1:251$271,1996$.

58. D. J. White and W. A. Take. GeoPIV: Particle Image Velocimetry (PIV) Software for Use in Geotechnical Testing. Technical Report CUED/D-SOILS/TR322, Geotechnical and Environmental Research Group, University of Cambridge, UK, 2002. 
59. D. J. White, W. A. Take, and M. D. Bolton. Soil Deformation Measurement using Particle Image Velocimetry (PIV) and Photogrammetry. Gotechnique, 53(7):619-631, 2003.

60. P. Wriggers. Nonlinear Finite Element Methods. Springer-Verlag Berlin Heidelberg, 2008.

61. D. L. Youngs. Time-Dependent Multi-Material Flow with Large Fluid Distortion, pages 273-285. In K. W. Morton und M. J. Baines (eds.): Numerical Methods for Fluid Dynamics. Academic Press, London, 1982.

62. O. C. Zienkiewicz, A. H. C. Chan, M. Pastor, B. A. Schrefler, and T. Shiomi. Computational Geomechanics - With Special Reference to Earthquake Engineering. John Wiley \& Sons, Chichester, 1999.

63. O. C. Zienkiewicz and R. L. Taylor. The Finite Element Method, 3 vols. Butterworth-Heinemann, 5th ed., 2000. 